\title{
Applying Classical and Intelligence Techniques for Digital Image Contrast Enhancement
}

\author{
Alyaa Q. Ahmed Taqi \\ Dr.alyaa@uomosul.edu.iq \\ College of Computer Sciences and Mathematics \\ University of Mosul, Iraq

\section{Received on: 30/03/2008}

\section{ABSTRACT} \\ Accepted on: 11/06/2008
}

Modern digital camera technology has produced huge services for the users from different ages and specifications .It made it easer to have images, but the user still needs to enhance those images, which have some problems when taken by the camera, for not applying enough light, as taking it in cloudy weather or on bright light or dark area or taking it from a far distance, all these reasons make the picture not clear having ambiguous details and colors. So, through this research we used some image contrast enhancement techniques to adjust the light for dark images, to make them have deep detail, sharp edges and better quality. Contrast problem is one of the most problems that face those who work on research field or normal users.

The aim of this research is to improve the contrast of images that have bad contrast using both classical techniques and intelligence techniques. Among intelligence techniques we chose the fuzzy logic methods, to have images contain better colors all over the image and make the images look brighter. By studying the classical and fuzzy logic methods, we proposed a method named (Fuzzy Hyperbolic Threshold), the proposed method gave very good results. We applied the methods on gray, colored images and on a video, and used (Matlab 7) to implement those methods.

Keywords: enhancement, intelligence techniques, contrast, brightness

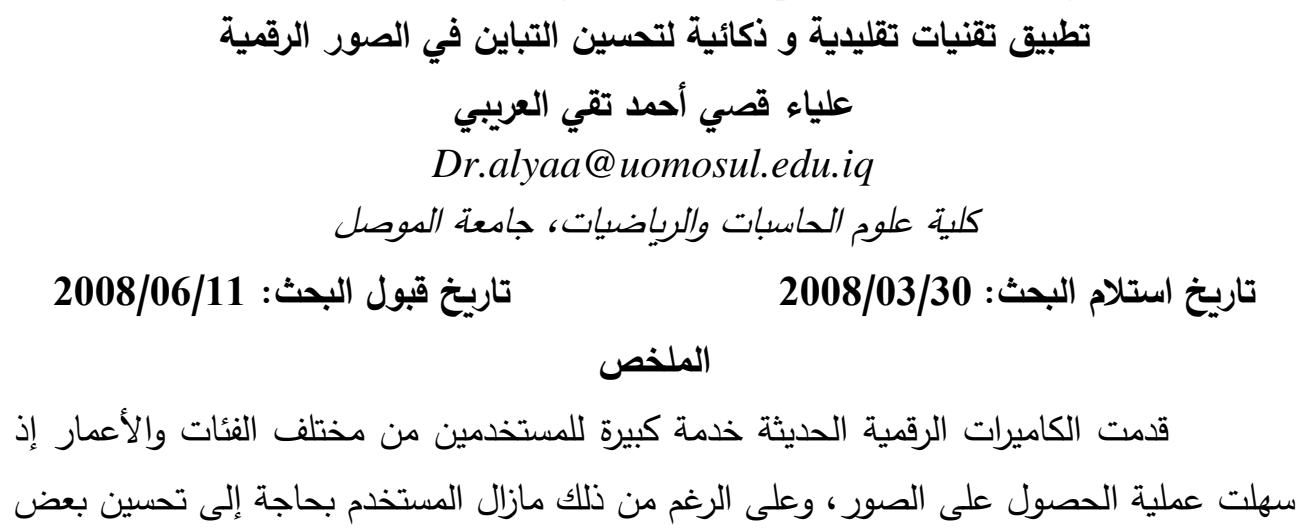


الصور التي يشوبها عدم الوضوح عند التقاط الصورة بسبب عدم توافر الإضاءة المناسبة كالجو

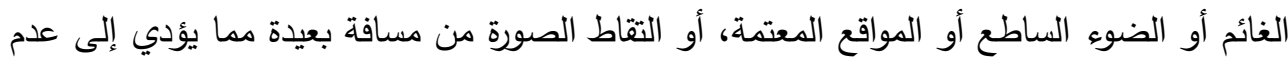

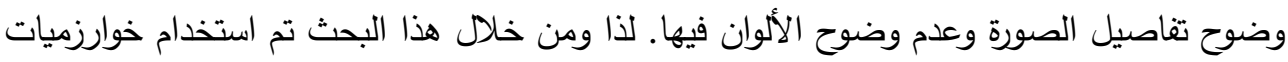
تحسين التباين في الصور (Contrast Enhancement) لما لها من أثر كبير في تعديل الإضاءة في الصور المعتمة، توضيح حوافها، توضيح معالمها وتحسين جودة الصورة. إن مسألة التباين السيئ في الصور من أكثر المشاكل التي تواجه المستخدمين أو الباحثين لاى عملهم على على صوض صور

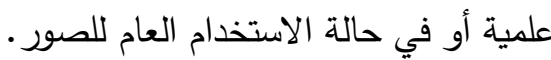
يهدف البحث إلى تحسين تباين الصور الرقمية ذوات التباين غير الجيد،والمقارنة بين

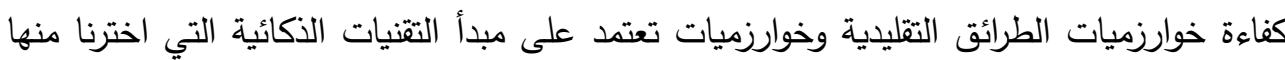
الطرائق المعتمدة على المنطق المضبب (Fuzzy logic)،وذلك للحصول على صورة يكون توزيع الألوان فيها توزيعاً جيداً، وجعل الصور أكثر بريقا. ومن دراسة خواص الطرائق التقليدية وطرائق المنطق المضبب المستخدمة في البحث، تم اقتراح طريقة لتحسين التباين في الصور سميت

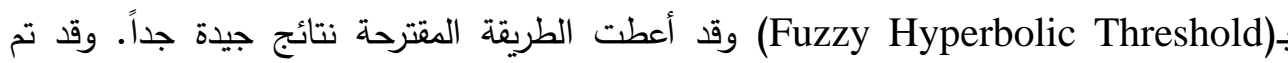
تطبيق البحث على صور ذات تدرج رمادي وصور ملونة وكذلك على فلم (movie) وتمت برمجة

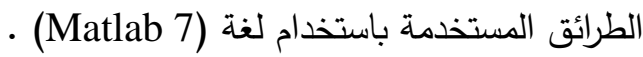

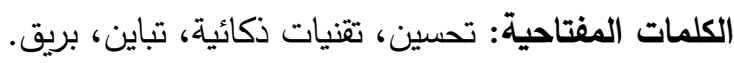

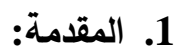

ازداد استخدام الصور الرقمية في السنوات الأخيرة نظرا لتوافر التكنولوجيا والأجهزة التي

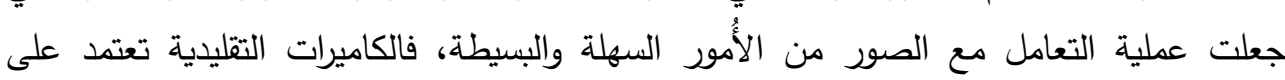

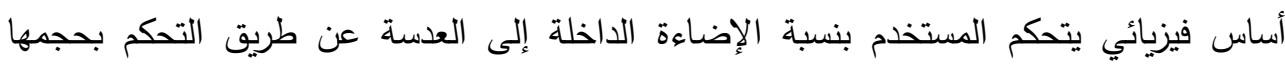
وباتجاه الضوء، أما الكاميرا الرقمية فلا تتطلب سوى دراية بسيطة من قبل المستخدم ومبدأ عملها لإصاءة يعتمد على تحويل الضوء إلى شحنات كهربائية، وتحويل الصورة إلى سلسلة من الأصفار والآحاد

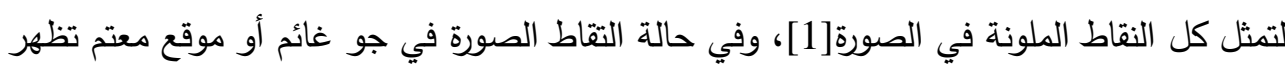

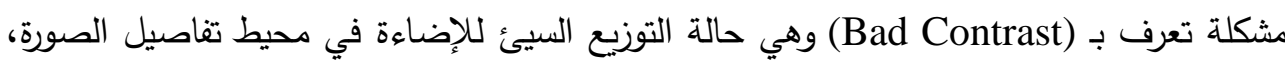

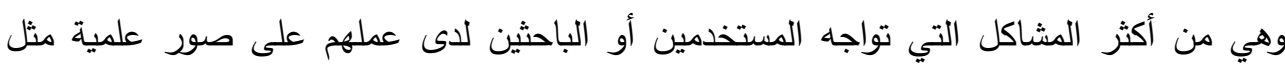

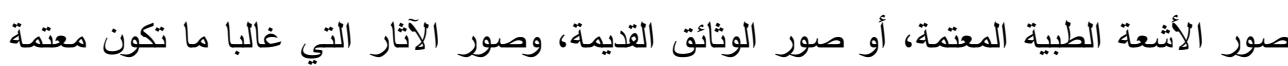

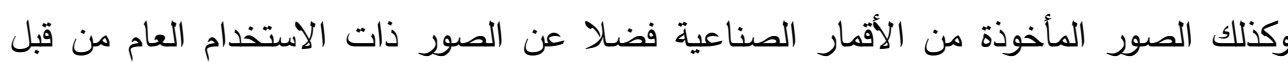




\section{2 أسباب الخلل في التباين:}

يُثير مصطلح التباين إلى مقدار الاختلاف بين الإضاءة المختلفة لعناصر الصورة إذ إذابن يعرف التباين (Contrast): "على أنه النسبة بين إضاءة الأجسام وإضاءة الأرضية التي تقع عليها الأجسام"، [10] إذ يعتمد التحس للتباين على التوزيع ألحيزي للمناطق المضيئة والمعتمة في التئي

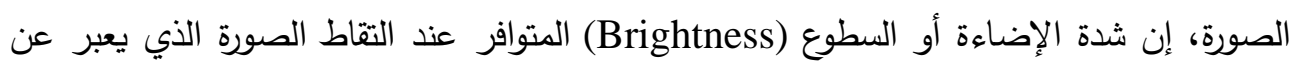
كمية الضوء المنعكس من أو المنتقل من خلال الكائن (Object)(الذي تلتقط صورته بواسطة الكاميرا من أهم الأُمور التي تؤثر في جودة الصورة [8] فإذا كان المستخدم (لا يملك خبرة في كيفية

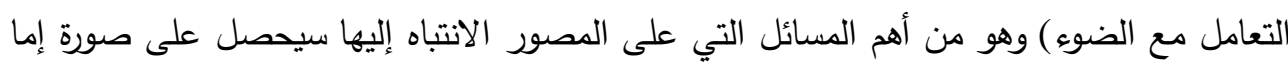

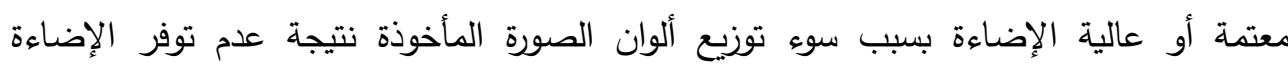

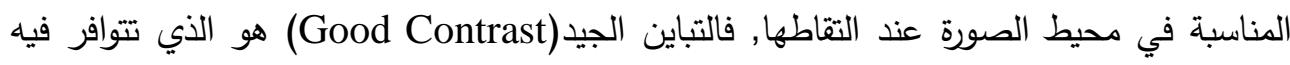

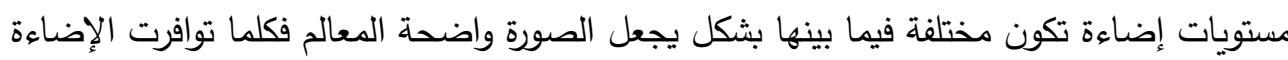

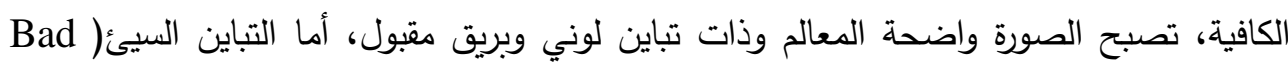
(Contrast

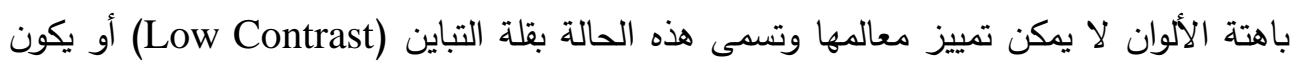
الاختلاف كبيراً إلى درجة يجعل مناطق من الصورة معتمة جداً ومناطق أُخرى ساطعة وتسمى هذه

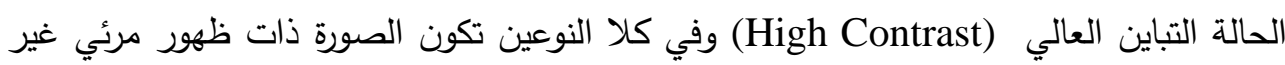
جيد مما أدى إلى ضرورة معالجة هذه المشكلة. 3.

يستجيب نظام الرؤية في عين الإنسان لمدى واسع من مستويات الإضاءة وهذه الاستجابة

(Dark

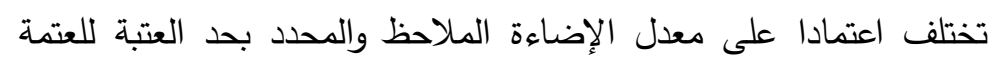
Threshold (Glare Limited) وحد للسطوع فالكثافات الضوئية التي هي أقل من حد العتبة للعتمة تكون معتمة جداً بحيث لا ثُرى أما الكثافات التي أكثر من حد السطوع تكون مضيئة جداً

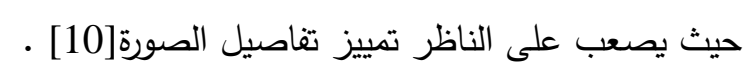

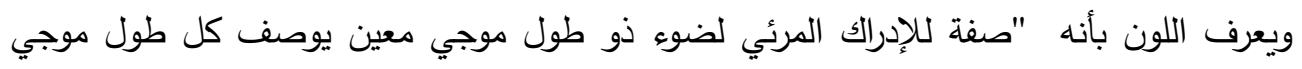

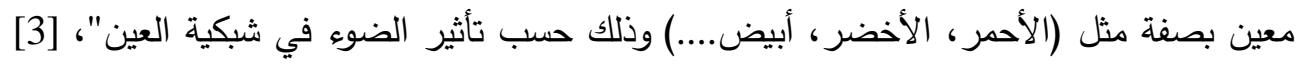

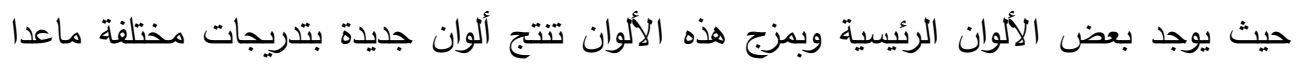

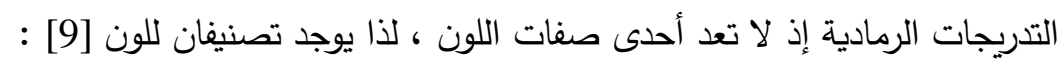


1. (Chromatic colors) : ويقصد أي لون ذي صبغة أي ألوان الطيف المعروفة ماعدا الأبيض

$$
\text { والأسود والتدريجات الرمادية. }
$$

2. (Achromatic colors) : (التي لا تعتبر لوناً نقياً ذا صبغة مثل )الأبيض والأسود والتدريجات الرمادية.

4. تمثيل الصور الرقمية (Digital Images Representation):

في جهاز الحاسوب تتم قراءة اللون من معرفة إحداثيات النقطة الضوئية في الصورة ،

وتمثل الصورة الرقمية مصفوفة ثنائية الأبعاد (Two Dimensional Array) من البيانات الرقمية

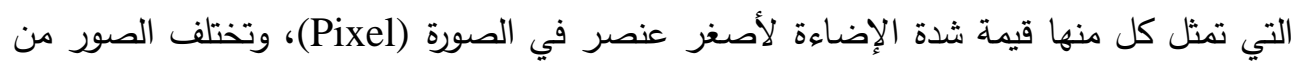

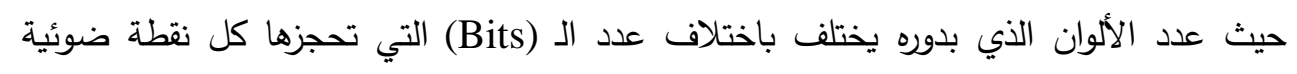
مثني الصورة، ويتم تمثيل قيمة اللون في الثاشات الرقمية حسب نظام (RGel)

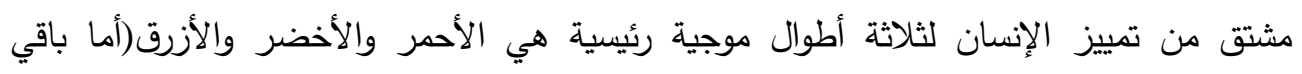

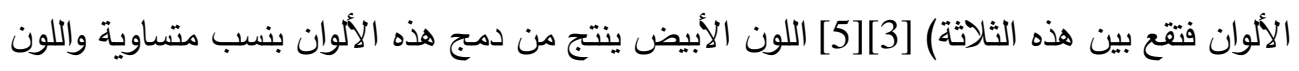

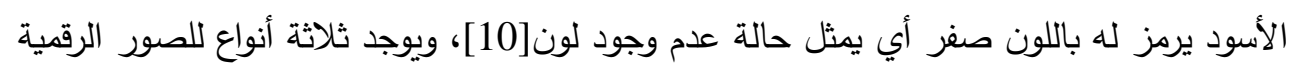

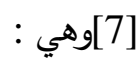

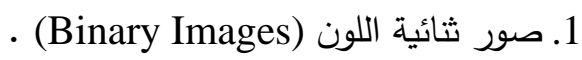

2. صور ذات التدرج الرمادي (Gray Level Images).

3. الصور الملونة (Colored Images) . 5. العوامل المؤثرة على تمثيل اللون :

تختلف قيمة اللون باختلاف ثلاثة عوامل رئيسية هذه العوامل تحدد اللون الذي يراه

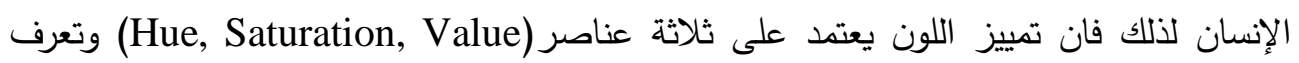

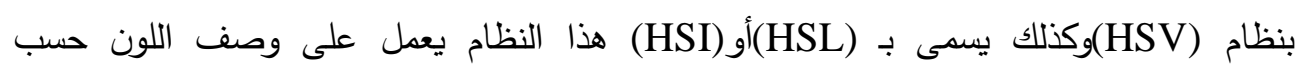

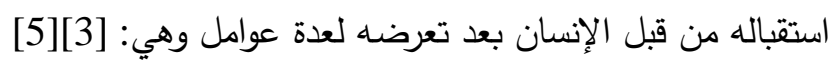

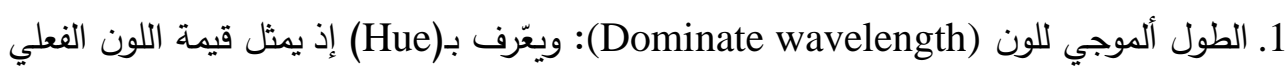

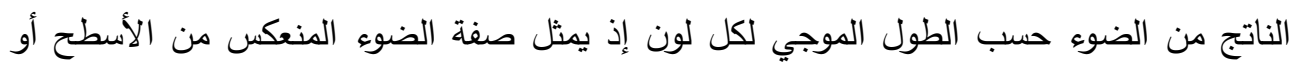
الأجسام. 
2. الإثباع (Saturation): يمثل درجة التثبع اللوني للون مثلا اللون الناتج من طول موجي معين

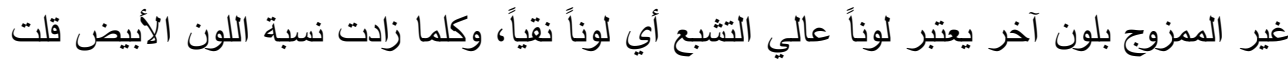
نسبة التثبع.

3. شدة اللون(Value, Lightness, Intensity): التسميات الثلاثة لها نفس الدلالة إذ تمثل قيمة البريق للون وهو مقياس لـقدار الضوء المنعكس من الأجسام أو مقدار الطاقة الناتجة من الأجسام

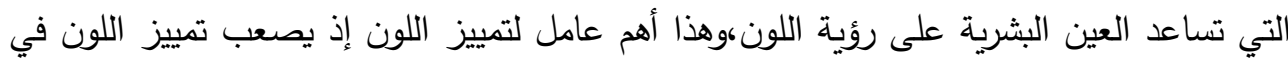

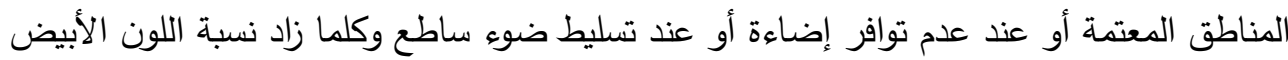
قل بريق اللون ومن ثم فقد خواصده، والثكل(1) يوضح هذه المفاهيم الثلاثة إذ يتغير (Hue) بشكل عمودي ونسبة التشبع تتغير بثكل قطري أما الإضاءة فتزادا كلما اتجهنا إلى الأعلى.

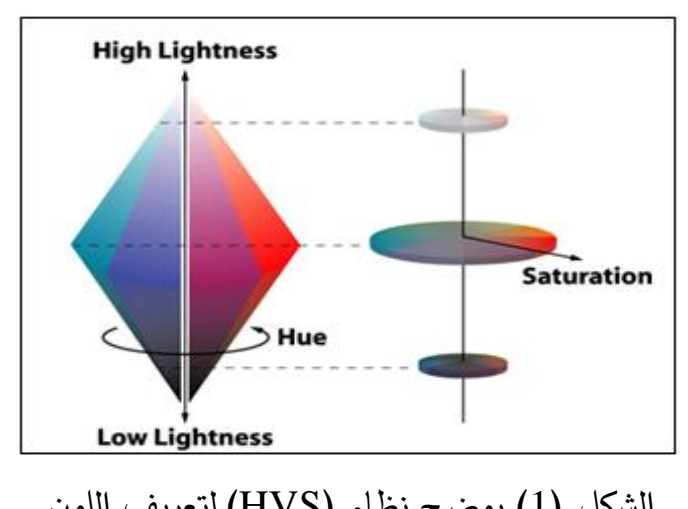

الثكل (1) يوضح نظام (HVS) لتعريف اللون

6. المنطق الضبب (Fuzzy Logic) :

طرحت المجموعات المضببة والمنطق المضبب من قبل العالم الأذربيجاني الأصل لطفي خليل زاده في عام (1965)، ومنذ ذلك الحين أصبحت طرائق المنطق الدضبب إحدى الأساليب

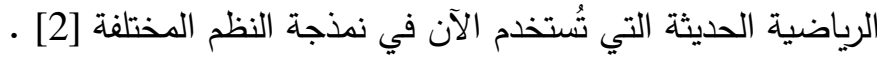

تتعامل نظرية المجموعات المضببة (Fuzzy Set Theory)مع المسائل التي تتضمن لا وثوقية لغوية نتيجة الغوض في بعض العبارات اللغوية مثل "قديم"، "عالِّ"، "طويل"، "حار"، ...الخ ,

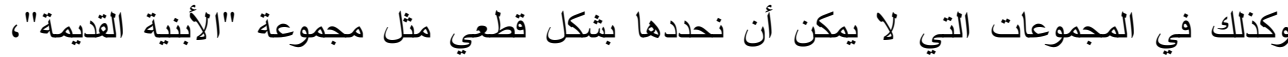

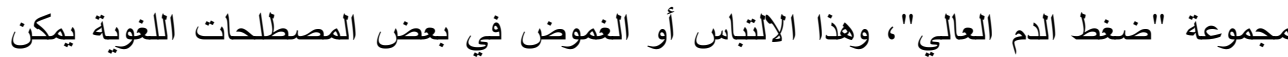
التعامل معه من خلال المجموعات المضببة وذلك بالتخلص من شكل الحدود التي تفصل العناصر التي تتنمي إلى المجموعة والعناصر التي لا تتنمي إليها عن طريق إعطاء صيغة قانونية للتعامل 
مع حالة عدم الدقة الحقيقية للعديد من المسائل وسنتاول فيما يلي تعريف بعض المفاهيم الأساسية

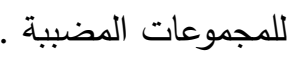

6.1 المجموعات الهشة والمجموعات المضببة (Crisp Set and Fuzzy Sets) :

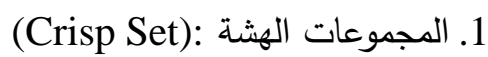

من المعروف أنه عند التعامل مع المجموعات الكلاسيكية (Classical Sets) ، تكون العناصر في

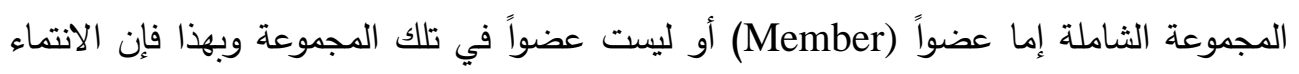
أو ما يسمى بالعضوية (Membership) للعناصر يكون هشاً أي يكون إما نعم أو لا،صواباً أو خطأ [2][9] وهذه الصفة هي التي تميز المجموعة الكلاسيكية ولذلك تسمى بمصنلح نظرية المجموعات المضببة بالمجموعة الهثة (Crisp Set) وكما موضح: مثلاً: لتكن (X) تمثل المجموعة الثاملة وإن(A) هي مجموعة هثة معينة، نلاحظ إن جميع (C) وكمات موضع:

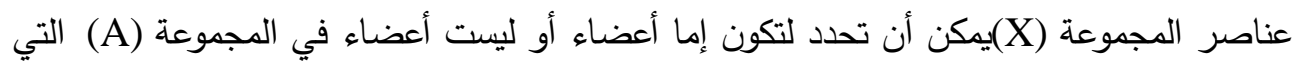

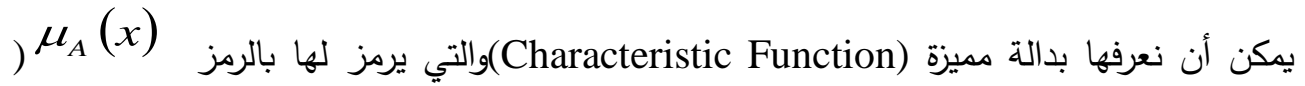

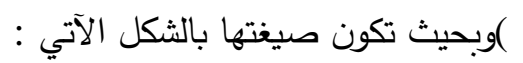

$\mu_{A}(x)\left\{\begin{array}{c}1 \Leftrightarrow x \in A \\ 0 \Leftrightarrow x \notin A\end{array}\right\}$

2. (Fuzzy Sets) : المجموعات المضببة

تعد المجموعات المضببة تعميماً للمجموعات الهشة وذلك بإعطاء درجة للعضوية لكل عنصر في المجموعة، وبهذا يمكننا تعريف المجموعة المضببة بأنها مجموعة جزئية من المجموعة

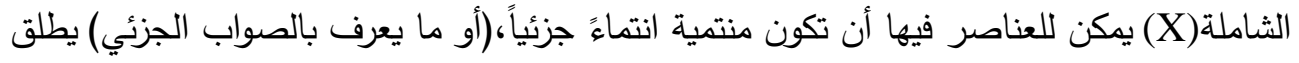
على درجة انتمائها درجة العضوية (Membership degree)والتي تكون أعداداً حقيقية تقع ضمن الفترة المغلقة [1,0] وتمثل درجة العضوية بالثكل الآتي:

$$
\mu_{\mathrm{A}}: \mathrm{X} \rightarrow[0,1]
$$

(Membership Function): 6.2

إن كل مجموعة مضببة (A) معرفة بدلالة مجموعة شاملة مناسبة(X)تعرف بواسطة دالة،

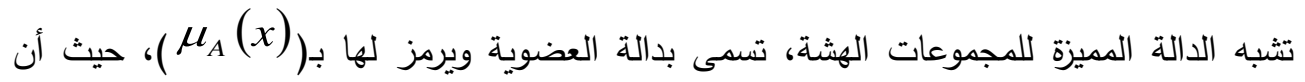

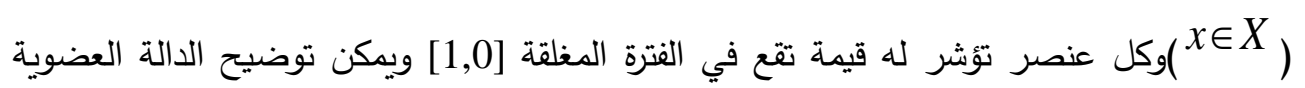
بالشكل الآتي : 


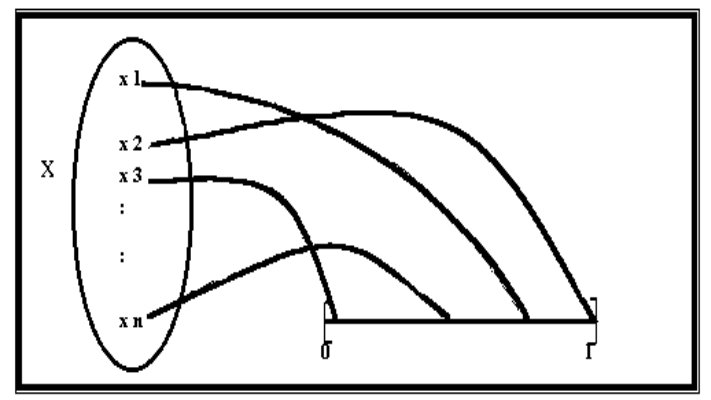

الثكل (2) يوضح التشيل العام لدالة العضوية

6.2.1 تمثيل الدوال العضوية (Representation of Membership Functions) :

يمكن تمثيل الدوال العضوية بأكثر من طريقة، وندرج في أدناه أكثر هذه الطرائق شيوعاً [2]:

1. التمثيل البياني(Graphical Representation): يعد هذا التمثيل من أكثر الطرائق شيوعاً في

الاستخدام، حيث تمنَّل دالة العضوية بمنحني معين يمكن اختياره حسب طبيعة المسألة .

2. التمثيل ألجدولي (Tabular Representation): يضم الجدول الممثل للمجموعة المضببة بـنية جميع العناصر في المجموعة الثاملة ودرجات العضوية المطابقة لها وتستخدم هذه الطريقة عندما نميز المجموعة المضببة لأعضاء المجموعة التي تكون مرتبطة مع درجات العضوية في

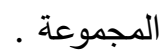

3. التمثيل الهندسي(Geometric Representation):لتكن (X)(مجموعة شاملة منتهية تضم)(n) من العناصر X $X=\left\{x_{1}, \ldots, x_{n}\right\}$ ، إن كل عنصر في (X) ممكن أن يُنظر إليه كإحداثي في

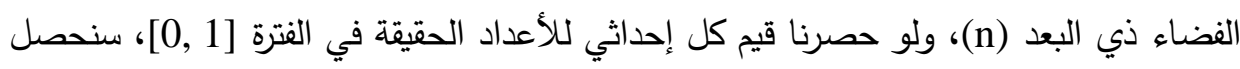
على مجموعة جزئية من الفضاء والتي تسمى مكعب الوحدة ذا البعد (n). 4. التمثيل التحليلي (Analytic Representation):عندما تكون المجموعة الثاملة غير منتهية

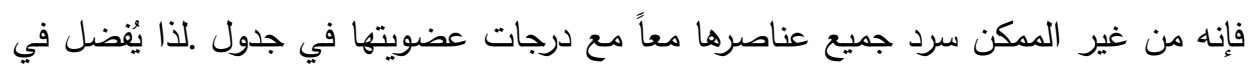
مثل هذه الحالات أن تمثّل دالة العضوية بشكل صيغة رئل رياضية معينة، ومن أشهر هذه الصيغ:

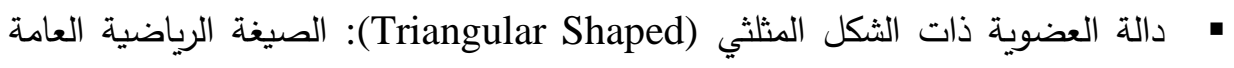
$\mu_{\mathrm{A}}(\mathrm{x})=\left\{\begin{array}{ccc}1-\frac{|\mathrm{x}-\mathrm{a}|}{\mathrm{c}} & ; & |\mathrm{x}-\mathrm{a}| \leq \mathrm{c} \\ 0 & ; & \text { otherwise }\end{array}\right\}$

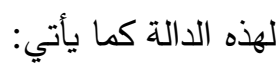


• دالة العضوية ذات الثكل شبه المنحرف (Trapezoidal Shaped):صيغتها كالآتي: (a) (a) (a) (T)

$\mu_{A}(x)=\left\{\begin{array}{ccc}\frac{(a-x)}{(a-b)} & ; & a<x \leq b \\ 1 & ; & b \leq x \leq c \\ \frac{(d-x)}{(d-c)} ; & c \leq x<d \\ 0 & ; & \text { otherwise }\end{array}\right\}$

• دالة العضوية ذات الثكل الجرسي : (Bell Shaped)،تسى أيضاً بالدالة الكاوزية (Gaussian Function)

$\mu_{A}(x)=e^{-\frac{(x-a)^{2}}{b}}$

والثكل (3) يوضح شكل دوال العضوية المذكورة :

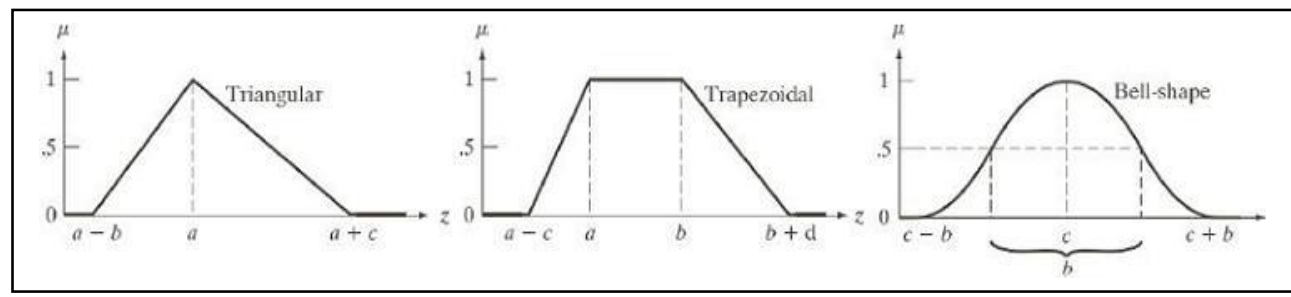

الثكل (3) يوضح أشكال بعض دوال العضوية

7. الطرائق التقليدية المستخدمة في تحسين التباين (Contrast Enhancement):

قمنا في بحثنا هذا باستخدام ثلاث طرائق تقليدية تعتبر من أبرز الطرائق المستخدمة في تحسين التباين في الصور ، كما استخدمنا طرائق تعتمد على المنطق المضبب وذلك لتوضيح الفرق

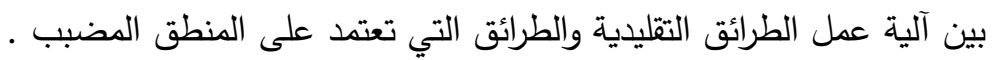
في كلا الأسلوبين يتم تحسين التباين في الصور الرقمية عن طريق معالجة كل عنصر (Pixel) بحيث تكون الصورة الناتجة ذات مدى أكبر من السابق أي تحوي على اختلاف لوني الوني أكثر ،إذ تعتمد

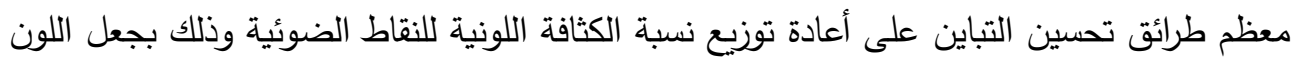
الغامق يبدو أغمق واللون الفاتح يبدو أفتح هذا التحويل يزيد الاختلاف بين عناصر الصورة وبذلك لكائل

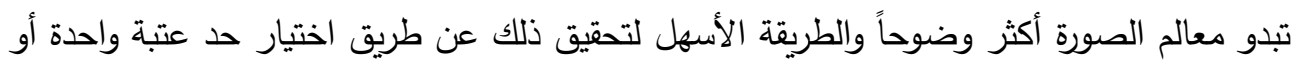
أكثر بحيث ان كل قيمة لونية أقل من قيمة حد العتبة تقلل أما إذا كانت أعلى فتزداد، لكنها لا تعتبر 
من الطرائق الكفوءة والمناسبة لكل أنواع الصور [4][8][10]، وسنتتاول فيما يأتي الطرائق التقليدية المستخدمة في هذا البحث وهي طريقة الددرج التكراري المتساوي، وطريقة نشر التباين وطريقة : مرشح تحسين التباين :

\section{1 المدرج التكراري المتساوي $($ Histogram Equalization):}

تعتبر هذه الطريقة من أكثر الطرائق استخداما في معالجة الصور، والمدرج التكراري هو

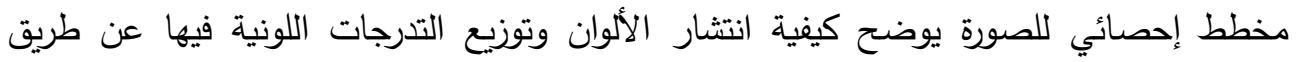

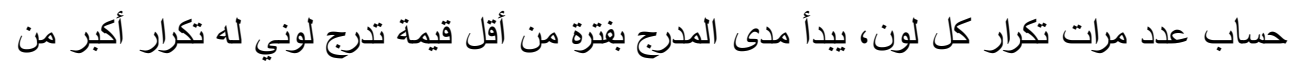

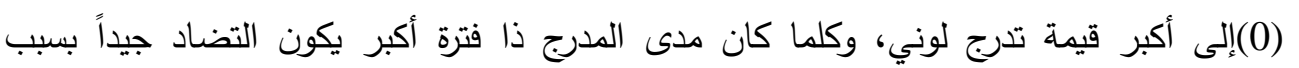
الاختلاف بين قيم المدرج، فتكون العلاقة بين انتثار القيم على الددرج وتباينها علاقة طردية

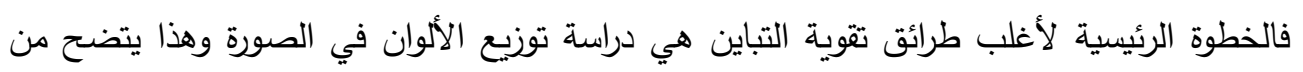
خلال المدرج التكراري[8] ،الهدف من هذه الطريقة هو الحصول على توزيع خطي ومتساوٍ للألوان على كل الصورة بدلاً من بقاء بعض القيم متركزة في موقع معين، إذ يتم اختيار دالة تحويل لتغيير

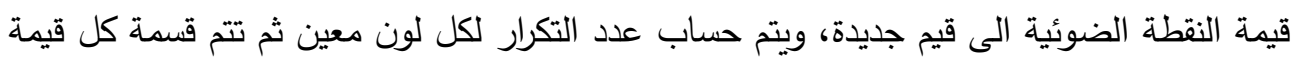
على مجموع الألوان في الصورة للحصول على قيم ألوان جديدة، ثم تحدد قيم جديدة لنسبة كل تكرار

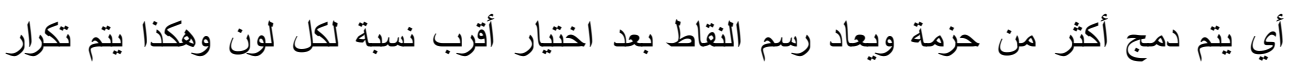

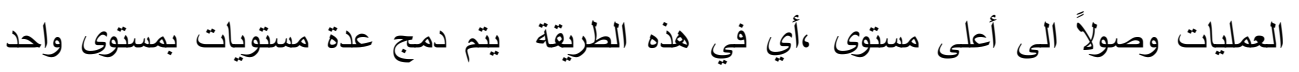

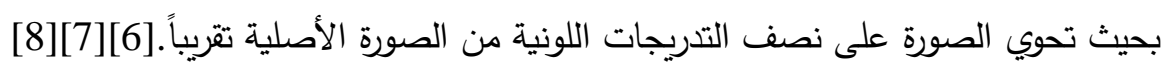
وندرج فيما يأتي خوارزمية المدرج التكراري المحسن:

Step 1: Read the origin image ( $\mathrm{g}$ ) of dimension $\mathrm{m}^{*} \mathrm{n}$

Step 2: Find the highest level in the image $(g(r, c)$ max $)$ then Set $\mathrm{i}=0$, Set total $=m^{*} n$

Step 3: Calculate the number of pixel $g(r, c)$ that have the same value where $n k(i)=g(r, c)$ (nk(i) hold the no.of pixel of specific value)

Step 4: For each pixel in the image, do the following :

- Find the average levels $\operatorname{pr}(\mathrm{i})=(\mathrm{nk}(\mathrm{i}) /$ total $)$

Step 5: for $i=2$ to max level in the image (first level remain with no change) begin:

- set the new level : sk(i)=pr(i-1)+p(i) (combine the levels)

- Set the new pixels to the new levels in the (sk(i))

- end 
Step 6: Show the new image

لكن تعتبر هذه الطريقة من أقل الطرائق كفاءة في معالجة التباين إذ تبدو الصورة

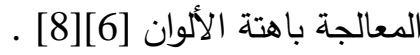

\section{2 طريقة نشر التباين (Contrast Stretching) :}

تعتبر من أبسط الطرائق المستخدمة لتقوية التباين ومفهوم هذه الطريقة هو إعادة نشر

التدرجات اللونية على كل الصورة للحصول على تباين أفضل من السابق [10]، وذلك بموازنة قيمة

كل (Pixel) مع أعلى وأقل قيمة لونية في الصورة وتمت برمجة هذه الطريقة بالخطوات الآتية :

Step 1: Read the origin image (g)

Step 2: Find minimum level in the image $\left(g(r, c)_{\min }\right)$ and the highest level in the image $\left(g(r, c)_{\max }\right)$

Step 3: For each pixel in the image, Calculate the new pixel $g^{\prime}(r, c)$ value through the following Mapping Function :

$$
\mathrm{g}^{\prime}(\mathrm{r}, \mathrm{c})=\left[\frac{\mathrm{g}(\mathrm{r}, \mathrm{c})-\mathrm{g}(\mathrm{r}, \mathrm{c})_{\text {min }}}{\mathrm{g}(\mathrm{r}, \mathrm{c})_{\max }-\mathrm{g}(\mathrm{r}, \mathrm{c})_{\min }}\right][\mathrm{MAX}-\mathrm{MIN}]+\mathrm{MIN}
$$

Step 4: Show the enhanced image ( $\mathrm{g}^{`}$ )

هنا تم حساب أعلى وأقل قيمة في الصورة ككل، ويمكن تطبيق الفكرة نفسها على جزء من الصورة

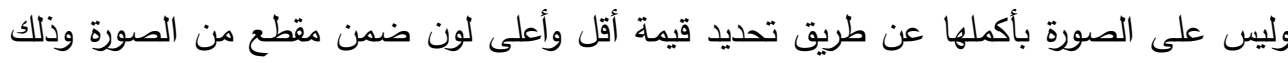

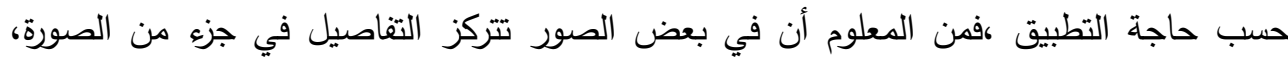
فيمكن إجراء تحسن التباين على هذا الجزء فقط[10]. وعلى الرغم من بساطة هذه الطريقة تعتبر

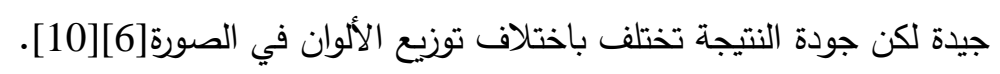

7.3 مرشح تحسين التباين (Adaptive Contrast Enhancement Filter) :

يعتبر استخدام المرشحات (Filters) من أكثر الطرائق استخداما في معالجة الصور ويطبق المرشح أما على كل عناصر الصورة أي تعتبر الصورة وحدة واحدة وتسمى هذه الطريقة

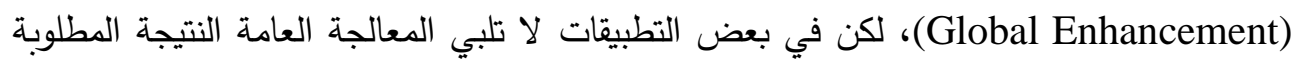

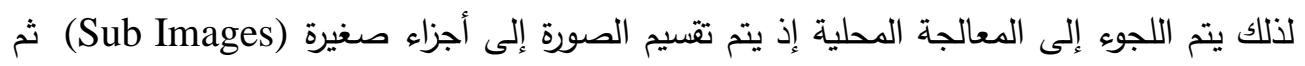

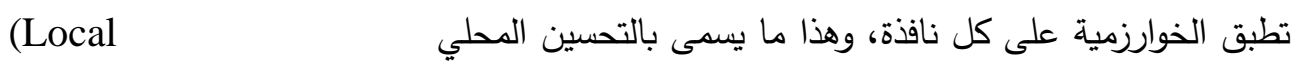

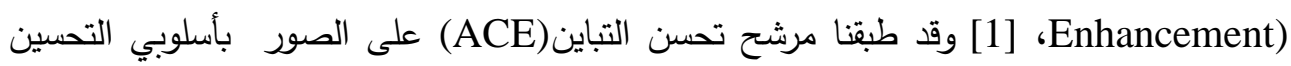

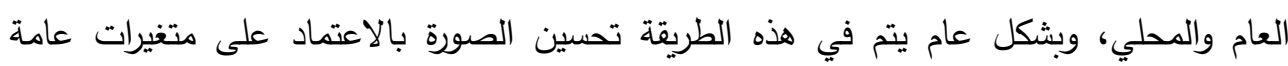

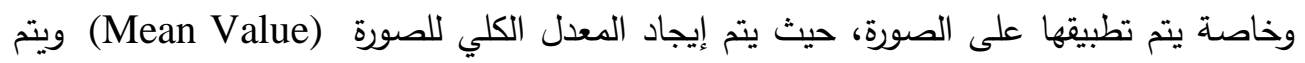




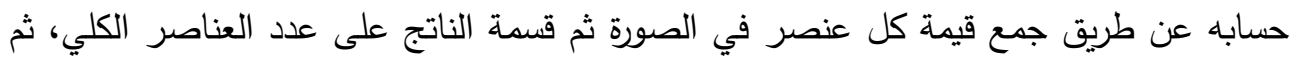

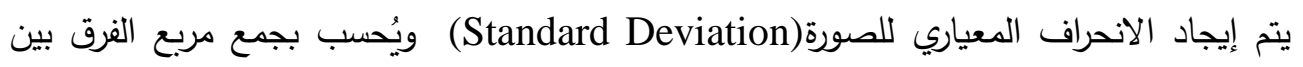

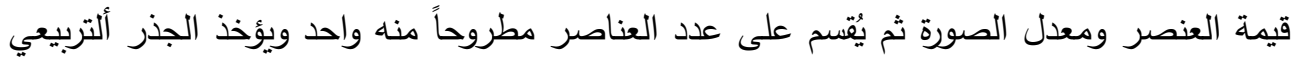

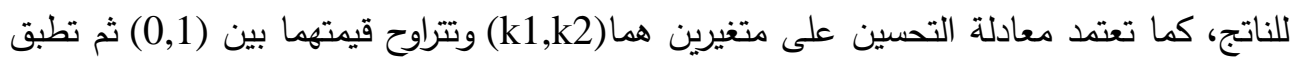

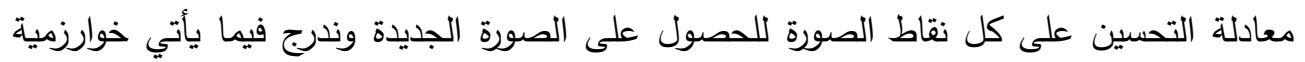
طريقة المرشح : - معند

Step 1: Read the original image ( $\mathrm{g}$ ) of dimensions $\mathrm{M} * \mathrm{~N}$

Step 2: Specify the parameters values:

- Specify the value of $(\mathrm{k} 1, \mathrm{k} 2)$ which its range from [0 1]

- Calculate the mean value $\left({ }^{M_{g}}\right)$ for the entire image where

$$
M_{g}=\frac{1}{m^{*} n} \sum_{(r, c) \in g} g(r, c)
$$

- Calculate the Standard Deviation value $\left({ }^{\sigma_{g}}\right)$ for the image where:

$$
\sigma_{g}=\sqrt{\frac{1}{\left(m^{*} n\right)-1} \sum_{(r, c) \in g}\left(g(r, c)-M_{g}\right)^{2}}
$$

Step 3: For each pixel in the image:

- Apply the enhancing filter (ACE) for each pixel $g(r, c)$ in the image, where:

$$
A C E=k_{1}\left[\frac{M_{g}}{\sigma_{g}}\right]\left[g(r, c)-M_{g}\right]+k_{2} M_{g}
$$

- Set the New Pixel Value $g(r, c)=\mathrm{ACE}$

Step 4: Show the new image

$$
\begin{aligned}
& \text { لقد طبقنا هذه الخوارزمية باستخدام التحسين العام والمحلي والفرق بينهما, أن الطريقة الثانية تتم } \\
& \text { باختيار حجم نافذة يمثل حجم مقطع محدد في الصورة الأصلية ويعامل كل مقطع كصورة، ثم يتم }
\end{aligned}
$$

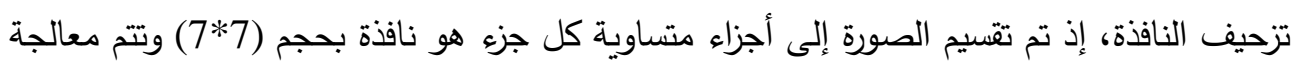

$$
\begin{aligned}
& \text { النوافذ بالتسلسل وذلك بتزحيف كل نافذة بمقدار (Pixel) واحدة، ويتم التزحيف من اليسار إلى هولى }
\end{aligned}
$$

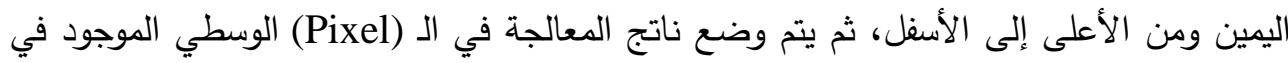

$$
\begin{aligned}
& \text { النافذة وهكذا إلى إن تتم معالجة كامل الصورة. }
\end{aligned}
$$




\section{8. طرائق المنطق المضبب (Fuzzy logic Techniques) :}

يستخدم المنطق المضبب في تحسين الصور الرقمية وذلك لإن بعض الصور تعاني من

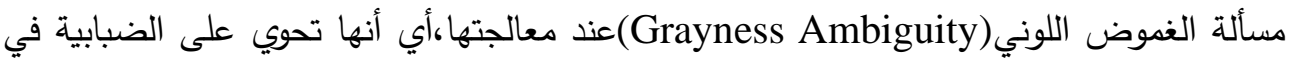
طبيعتها، فعند معالجة الألوان مثلا، يكون السؤال هل أن القيمة اللونية الحالية تصبح أدكن أم أسطع

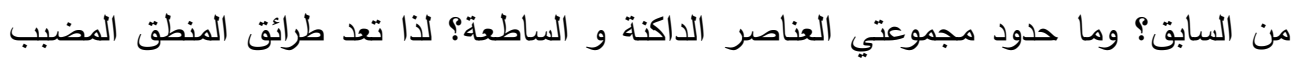
الأنسب في معالجة هذه المسائل، وتختلف طرائق المنطق المضبب في معالجة مسألة معينة في كيفية اختيار دالة عضوية مناسبة للحصول على النتائج المطلوبة، لكن تشترك الطرائق المضببة في في فئي

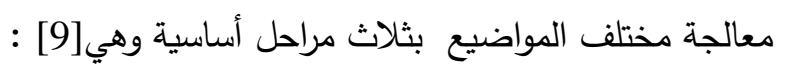

1. تضبب الصورة (Image Fuzzification): يتم في هذه المرحلة تحويل عناصر الصورة التي تُعتبر قيماً هشة إلى قيم مضببة باستخدام دوال العضوية حيث يُعطى لكل عنصر درجة

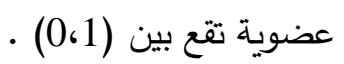

2. تحديث قيم دوال العضوية (Membership Modification):بعد الحصول على درجة

$$
\text { العضوية يتم تحديثها بقيمة مناسبة لنوع المعالجة المراد تطبيقها على الصورة }
$$

3. زوال الضبابية (Image Defuzzification): تُعتبر آخر مرحلة حيث يتم فيها تحويل القيم

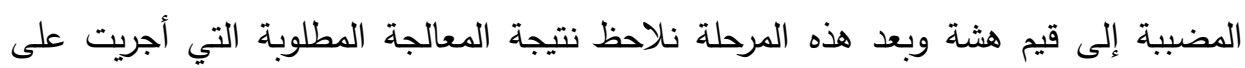
الصورة، والثكل (4) يوضح مراحل معالجة الصور باستخدام المنطق المضبب.

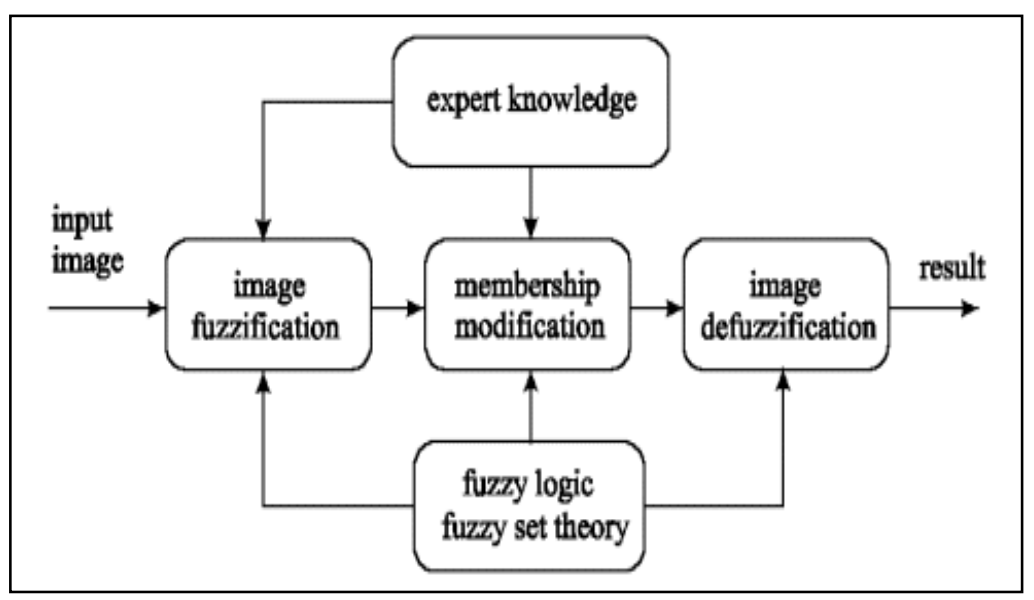

الثكل (4) يوضح مراحل معالجة الصور باستخدام المنطق المضبب

يتم اختيار دالة العضوية أو تصميمها حسب التطبيق المطلوب، وبالنسبة إلى تحسين التباين تم استخدام دالة عضوية تُعطي للعناصر الداكنة درجة انتماء قريبة من(0) أي لا تتنمي إلى الى 
لعناصر الساطعة، وتزداد هذه الدرجة بصورة تدريجية إلى أن تصل إلى (1) للعناصر الساطعة، أما باقي العناصر فتأخذ درجة انتماء بين(0,1) أي تتنمي انتماءً جزئياً إلى المجموعة والثكل الآتي يوضح نتيجة معالجة التباين في صورة ذات تدرج رمادي و المبدأ نفسه يطبق على صورة ملونة.

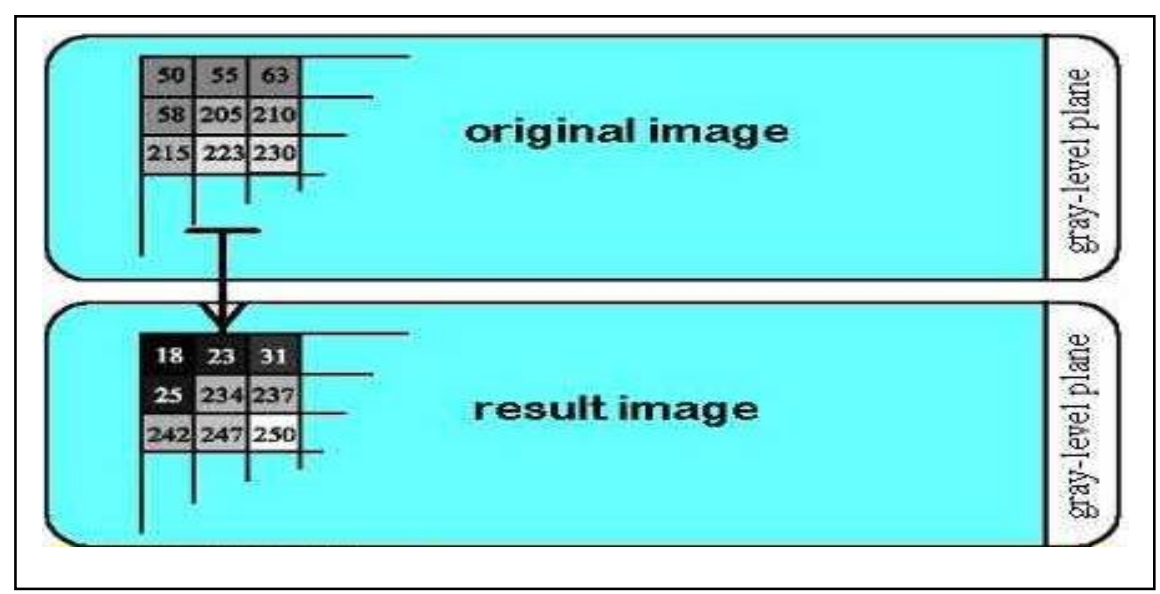

الثكل (5) يوضح عملية تحسين التباين في الصور باستخدام المنطق المضبب

8.1 الطرائق المعتمدة على المنطق المضبب (Fuzzy logic methods) :

تم استخدام ثلاث من الطرائق المضببة لتحسين التباين وهي طريقة تقوية التباين باستخدام

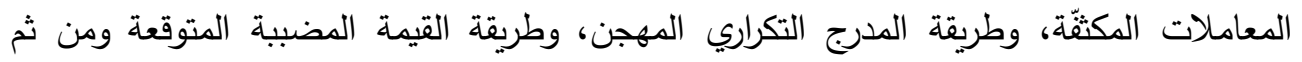
الطريقة المقترحة وهي طريقة حد العتبة المهنة المضببة:

8.1.1 طريقة تقوية التباين باستخدام المُعامِلات المُكثِفة(Intensification Operator): يُستخدم لتحسين التباين بنوعيه الواطئ والعالي بعض المعاملات التي تتحكم بقيمة كل نقطة ضوئية بالصورة الناتجة ،في هذه الطريقة لدينا المتغيران (Fe,Fd) اللذان يتحكمان بنتيجة

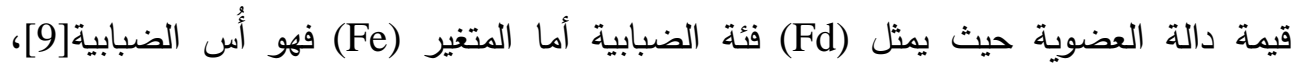
وتتضمن الخطوة الأولى تحديد معادلة درجة العضوية وتحديد كل من (Fe,Fd)وبعد أختيار قيمة درجة العضوية المناسبة لكل نقطة ضوئية، يتم تحديث درجة العضوية التي تم الحصول عليها من الخطوة الأولى حسب معادلة معينة، ثم يتم حساب القيمة الجديدة للا(Pixel) بموازنة القيمة الضبابية التي حصلنا عليها (بعد تحديث دالة العضوية) مع قيمة أعلى(Pixel) في الصورة الأصلية، وندرج فيما يأتي خوارزمية هذه الطريقة:

Step 1: Read the original image ( $g$ ) of dimensions $M^{*} N$ 
Step 2 :Initialize the parameters :

- Find the Maximum level in the image(g max)

- Specify the value of $(\mathrm{Fe})$ which its ranges from [0 1]

- Specify the value of (Fd) which its ranges from [10 100]

- Specify the membership $u(r, c)$ function which is :

$$
u(r, c)=\left[1+\frac{g_{\max }-g(r, c)}{F d}\right]^{-F e}
$$

Step 3 : for each Pixel $g(r, c)$ in the image apply Steps 3,4 and 5:

- Calculate the membership value $u(r, c)$ for $g(r, c)$

Step 4: Modify the membership $u^{\prime}(r, c)$ value as follows:

$$
u^{\prime}(r, c)= \begin{cases}2 \cdot[\mu(r, c)]^{2} & 0 \leq \mu(r, c) \leq 0.5 \\ 1-2 \cdot[1-\mu(r, c)]^{2} & 0.5<\mu(r, c) \leq 1\end{cases}
$$

Step 5: Get the new pixel value $g^{\prime}(r, c)$ as follows :

$$
g^{\prime}(r, c)=g_{\max }-F d\left[\left(\mu^{\prime}(r, c)\right)^{-\frac{1}{F e}}-1\right]
$$

Step 6: Show the processed image (g')

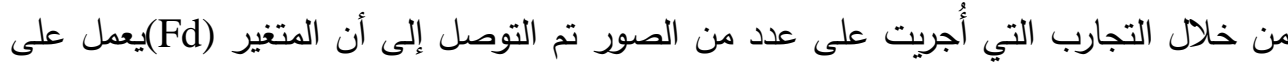

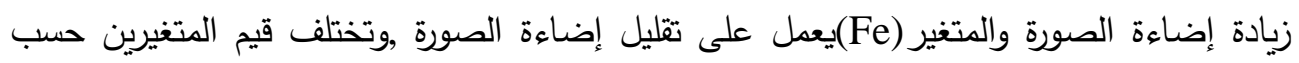
نوع التباين، فالصور ذات التباين الواطئ والتي تكون ذات ألوان باهتة تحتاج إلى قيمة (Fe)عالية

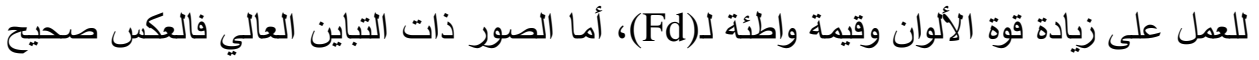
8.1.2 طريقة المدرج التكراري المهجن (Fuzzy Histogram Hyperbolization) :

للتخلص من مساوئ طريقة المدرج التكراري المتساوي في عمليات تحسن الصور اقترح العالم (Frei) طريقة (Histogram Hyperbolization) حيث أعتمد على قانون (Weber) الخاص بالإضاءة[6][9] فقد وجد هذا العالم أن العلاقة بين البريق(Brightness) المستقبل من

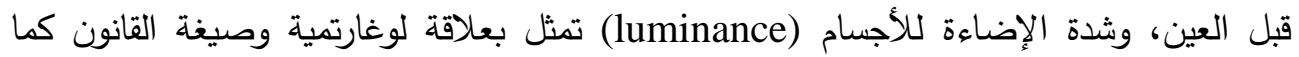
Brightness $=\log ($ Light Intensity + Contrast $)$ 
أي أن قيمة البريق المستقبل من العين تزداد بشكل لوغاريتمي لشدة الإضاءة التي تحددها العين

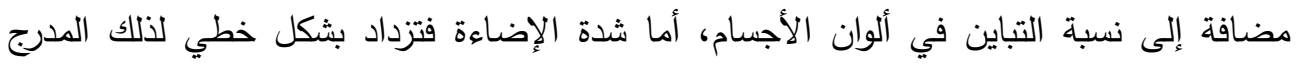

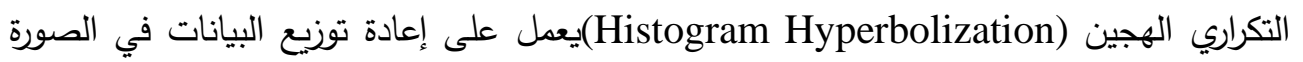
بحيث تصبح الصورة الناتجة ذات توزيع خطي لمستويات الإضاءة[4][6]، يتم في هذه الطريقة تقسيم التدرجات اللونية إلى ثلاث حزم ، وقد تم تكريس الصفة المميزة للمدرج التكراري المهجن لخدمة المنطق المضبب لتحسين التباين في الصور وذلك عن طريق تعديل القيم المضببة بشكل لوغاريتمي [4] وذلك حسب الخطوات الآتية :

في الخطوة الأولى يتم تصميم دالة عضوية وقد تم استخدام دالة عضوية تُعتبر تحديثاً للدالة ذات الثكل شبه المنحرف، حيث تُعطي درجة عضوية(0)للعناصر التي تمتلك قيمة أقل من (100) ودرجة عضوية (1) للعناصر التي تمتلك أكبر من (200)ودرجة عضوية بين (0,1) للعناصر التي تصني تمتلك قيمة بين (100,200)، أي يتم في هذه الطريقة تخطيط ألوان الصورة إلى ثلاث فئات،ثي يتم حساب قيمة دالة العضوية لكل نقطة،بعد ذلك يتم تحديث كل درجة عضوية مناسبة ولتكن م ، ثم تتم إزالة الضبابية لحساب القيم الجديدة للصورة بثكل لوغاريتمي والخوارزمية الآتية توضح عمل هذه الطريقة :

Step 1: Read the original image ( $g$ ) of dimensions $\left(M^{*} N\right)$

Step 2: initialize the parameters :

- Find the Maximum level(g max) in the image

- Specify the value of fuzzifier $(\beta)$ which its ranges from [0 1]

Step 3 : for each Pixel $g(r, c)$ in the image apply step 3,4 and 5

Calculate the membership value $u(r, c)$ from the follows :

$$
u(r, c)=\left\{\begin{array}{lr}
0 & \text { if } g(r, c) \leq 100 \\
h & \text { if } 100 \leq g(r, c) \leq 200 \\
1 & \text { if } g(r, c) \geq 200
\end{array}\right\}
$$

where $\mathrm{h}=(0.01 * \mathrm{~g}(\mathrm{r}, \mathrm{c}))-1$

Step 4 : Modify the membership value where ${ }^{u^{\curlywedge}(r, c)}={ }^{u(r, c)} \wedge \beta$

Step 5 : Set the new pixel value $g^{\prime}(r, c)$ as follows :

$$
\mathrm{g}^{\prime}(\mathrm{r}, \mathrm{c})=\left(\frac{\mathrm{L}-1}{\mathrm{e}^{-1}-1}\right) \cdot\left[\mathrm{e}^{-(\mathrm{u}(\mathrm{r}, \mathrm{c}))^{\beta}}-1\right]
$$

Step 6 : Show the resulted image 
8.1.3 طريقة القيمة المضببة المتوقعة (Fuzzy Expected Value) :

تعتمد هذه الطريقة على اختيار قيمة من قيم للصورة لكي تزحّف بقية القيم وتتنتر حولها

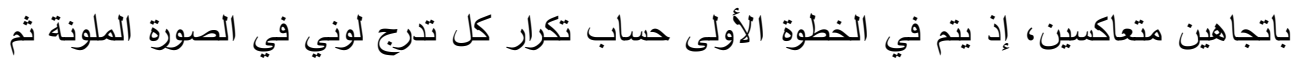

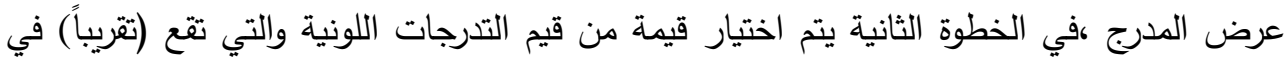
منتصف مدى المدرج ،بعد ذلك يتم حساب المسافة بين القيمة المختارة وقيم التدرجات اللونية في

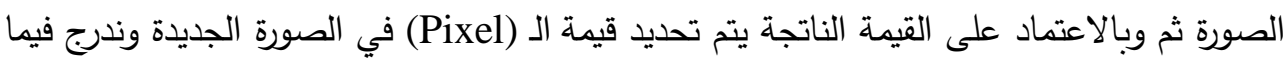
يأتي الخوارزمية المستخدمة في برمجة هذه الطريقة :

Step 1 :Read the original image ( $\mathrm{g}$ ) of dimensions $\left(\mathrm{M}^{*} \mathrm{~N}\right)$

Step 2 :Generate image histogram then find fuzzy expected value(FEV)

Step 3 :For each element in the image apply the following:

- Calculate the distance $\mathrm{D}(\mathrm{r}, \mathrm{c})$ from the pixel and the (FEV) :

$$
D(r, c)=\sqrt{\left|(F E V)^{2}-(g(r, c))^{2}\right|}
$$

- Set the new pixel value $g^{\prime}(r, c)$ as the following

$$
\begin{array}{lr}
g^{\prime}(r, c)=\max (0, F E V-D(r, c)) & \text { if } g(r, c)<F E V \\
g^{\prime}(r, c)=\min (L-1, F E V+D(r, c)) & \text { if } g(r, c)>F E V \\
g^{\prime}(r, c)=F E V & \text { otherwise. }
\end{array}
$$

Step 4 : Show the resulted image ( $g^{\prime}$ )

كما نلاحظ إن هذه الخوارزمية لا تحتاج إلى عملية تحديث لدالة العضوية، إذ يتم حساب المسافة بين قيمة كل نقطة ضوئية وبين القيمة المضببة المتوقعة التي سيتم نشر قيمتها على بقية النقاط الضوئية بعد موازنتها ومقارنة كل نقطة ضوئية بالقيمة المضبية المتوقعة .

\subsection{4 طريقة حد العتبة المهنة المضببة (Fuzzy Hyperbolic Threshold) :}

بعد الدراسة والاطلاع على عدة طرائق لتحسين التباين في الصور، نقترح طريقة وذلك بالاستفادة من ميزات الطرائق الجيدة سواءً التقليدية أو الذكائية، فكما ملاحظ فالئ لهان معظم الطرائق

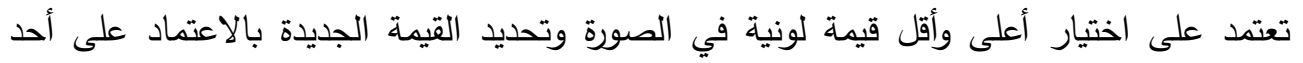

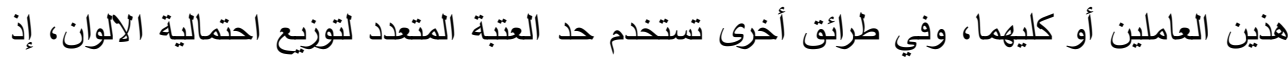

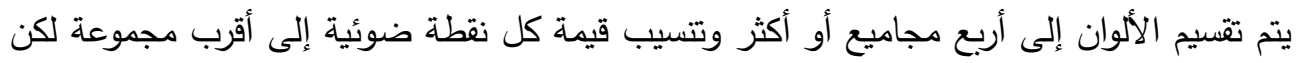

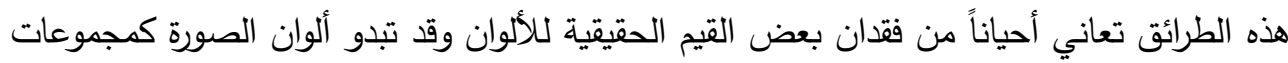

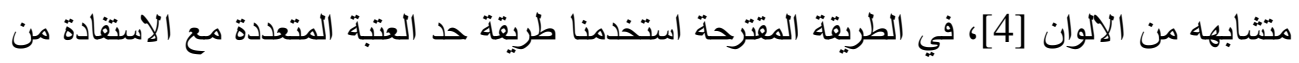


أسلوب طريقة نشر القيم (Stretch) التي تعمل على أخذ أعلى وأقل قيمة في الصورة لكن أضفنا إليها القيمة المتوسطة التي يكون لها تأثير كبير في الصورة وذلك حسب توزيع الألوان فيها، وذلك

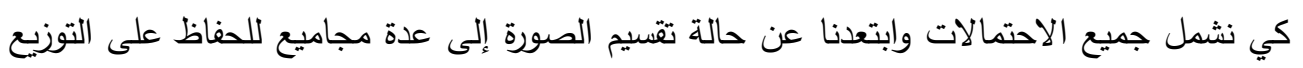
الفعلي للألوان، في طريقتنا تتم مقارنة كل نقطة ضوئية في الصورة مع أقل قيمة والقيمة المتوسطة

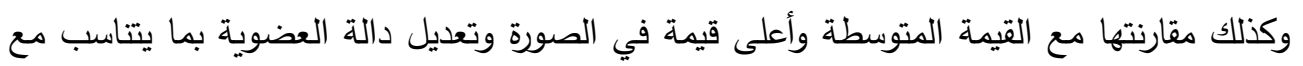

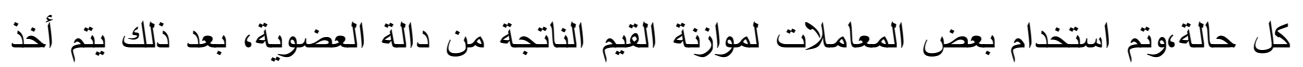

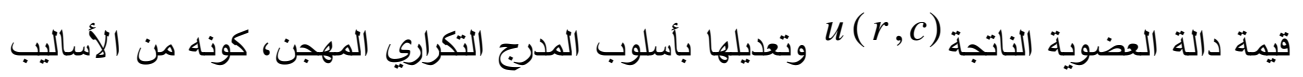
الجيدة في الحفاظ على قيمة اللون في الصور التي تعاني من مشاكل سوء الإضاءة [4][6][10]

$$
\text { وفيما يأتي توضيح للخوارزمية : }
$$

Step 1: Read the original image $(\mathrm{g})$ of dimensions $\left(\mathrm{M}^{*} \mathrm{~N}\right)$

Step 2: initialize the parameters :

- Find the Maximum (g max), Minimum (g min ) and the middle level value $(\mathrm{g}$ mid $),(\mathrm{g}$ mid $=(\mathrm{g} \max +\mathrm{g} \min ) / 2)$ in the image $(\mathrm{g})$

- Specify the value of fuzzifier $(\beta)$ which its ranges from [0 1]

Step 3: for each Pixel in the image do Steps 3,4 and 5:

- Calculate the membership value $u(r, c)$ as the follows :

If $(\mathrm{g}(\mathrm{r}, \mathrm{c})>=\min )$ and $(\mathrm{g}(\mathrm{r}, \mathrm{c})<\mathrm{mid})$ $\left.u(r, c)=0.5^{*}(\mathrm{~g}(\mathrm{r}, \mathrm{c})-\mathrm{g} \min ) /(\mathrm{g} \operatorname{mid}-\mathrm{g} \min )\right)^{\wedge} 2$ else if $(\mathrm{g}(\mathrm{r}, \mathrm{c})>=\mathrm{g}$ mid $)$ and $(\mathrm{g}(\mathrm{r}, \mathrm{c})<=\mathrm{g}$ max $)$

$$
\left.u(r, c)=1-(2 *(\mathrm{~g}(\mathrm{r}, \mathrm{c})-\mathrm{g} \max ) /(\mathrm{g} \max -\mathrm{g} \min ))^{\wedge} 2\right) ;
$$

Step 4: Modify the membership value where ${ }^{u^{`}(r, c)}={ }^{u(r, c) \wedge} \beta$

Step 5: Set the new pixel value $g^{\prime}(r, c)=u^{\prime}(r, c) * g(r, c)$

Step 6: Show the resulted image ( $\left.g^{\prime}\right)$.

\section{9}

لقد قارنا النتائج التي حصلنا عليها مع صورة تمت معالجتها ببرنامج (Photoshop)

الذي يعتبر من أقوى البرامج الخاصة بمعالجة الصور وتحسينها ،وقد رفقنا الصورة المحسنة بهذا مئا البرنامج للمقارنة مع الطرائق التي تمت برمجتها وملاحظة الفرق من خلال، شكل الصورة الناتجة

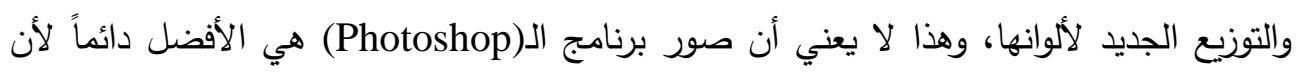

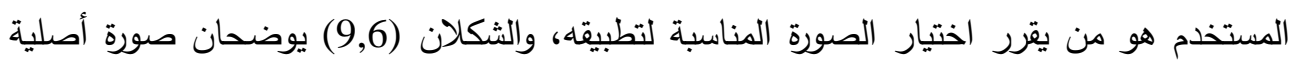
ذات تدرج رمادي وصورة أشعة طبية على التوالي، والثكلان (10,7) يوضحان النتائج التي حصلنا 
عليها عند تطبيق الطرائق التقليدية أما الثكلان $(11,8)$ فيوضحان نتائج تطبيق طرائق المنطق المضبب، والثكل (12) يوضح صورة ملونة ، والثكلان (14,13) يوضحان نتيجة تطبيق الخوارزميات عليهما، وتتم معاملة الصورة الملونة كثلاث صور إذ يتم عزل كل لون أساسي(RGB) من النقطة الضوئية على حدة، وتطبيق الخوارزميات على كل جزء لوني (RGB) ثم طباعة قيمة النقطة الضوئية الملونة الجديدة الناتجة من التطبيق بعد دمج الألوان الرئيسية الثلاثة لكل نقطة ضوئية.

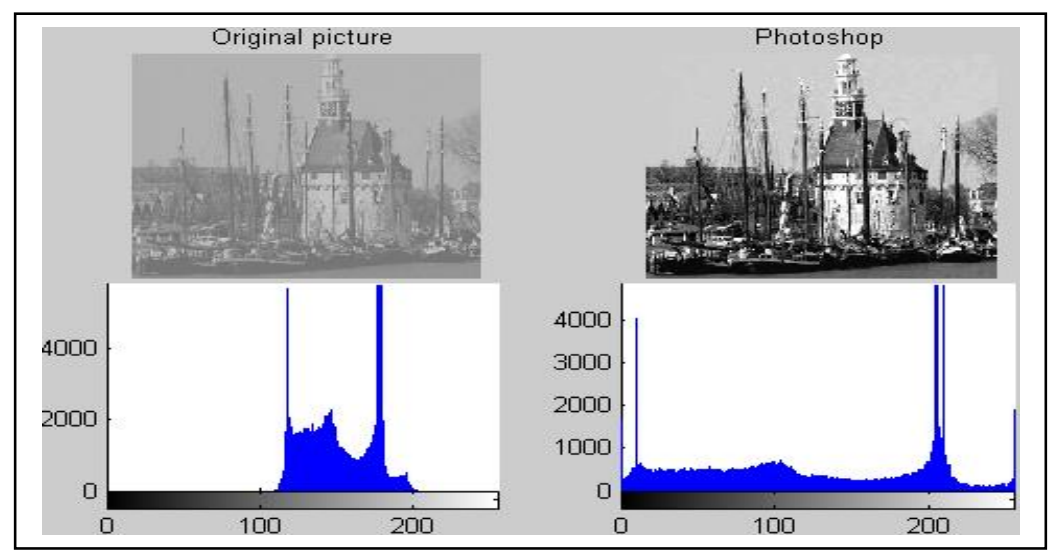

الثكل (6) يوضح الصورة التي ستتم معالجتها والصورة الناتجة من استخدام برنامج (Photoshop)

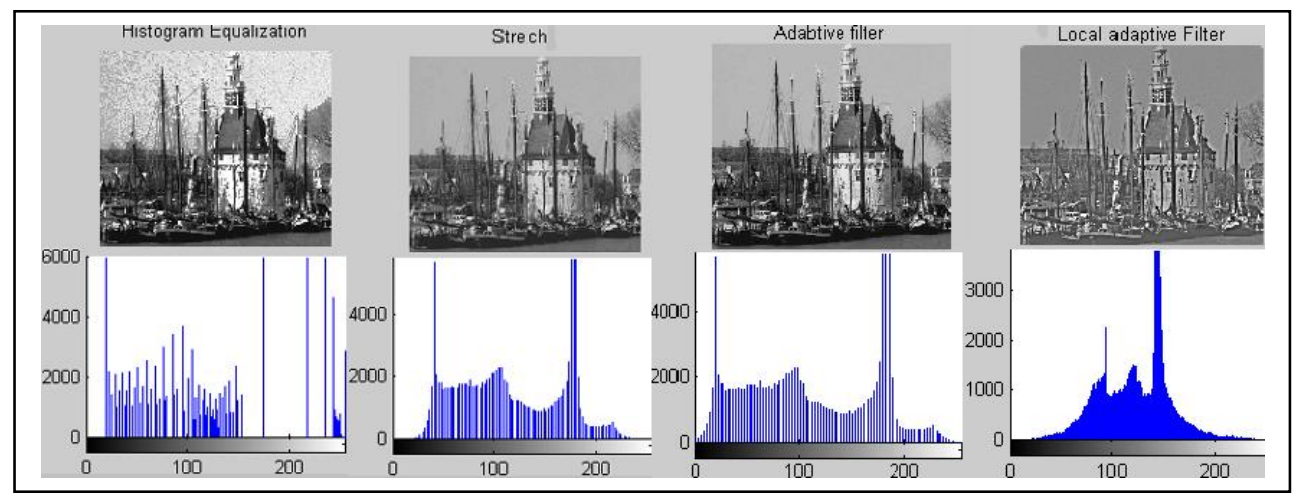

الثكل (7) يوضح الصورة الناتجة من عملية تحسين التباين باستخدام الطرائق التقليدية 


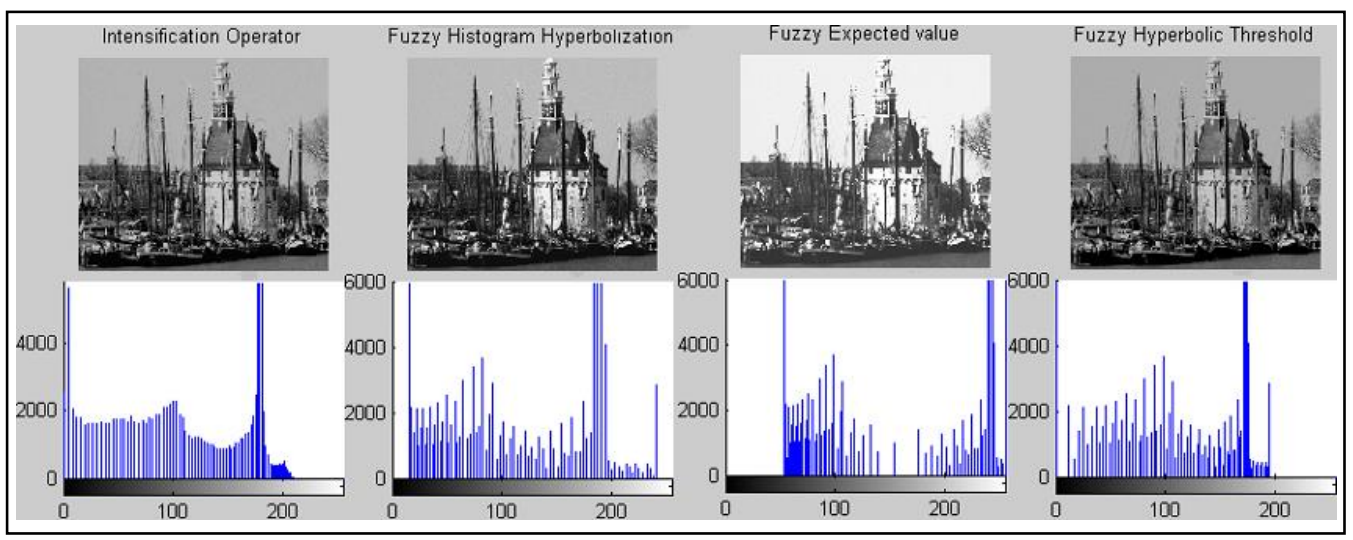

الثكل (8) يوضح الصورة الناتجة من عملية تحسين التباين باستخدام طرائق المنطق المضبب

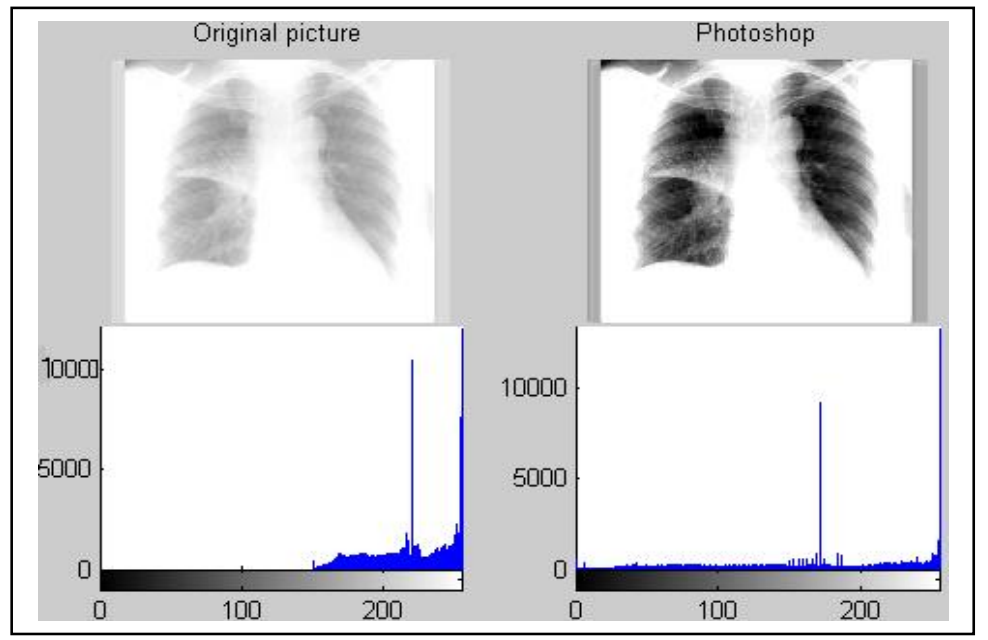

الثكل (9) يوضح الصورة التي ستتم معالجتها والصورة الناتجة من استخدام برنامج (Photoshop)

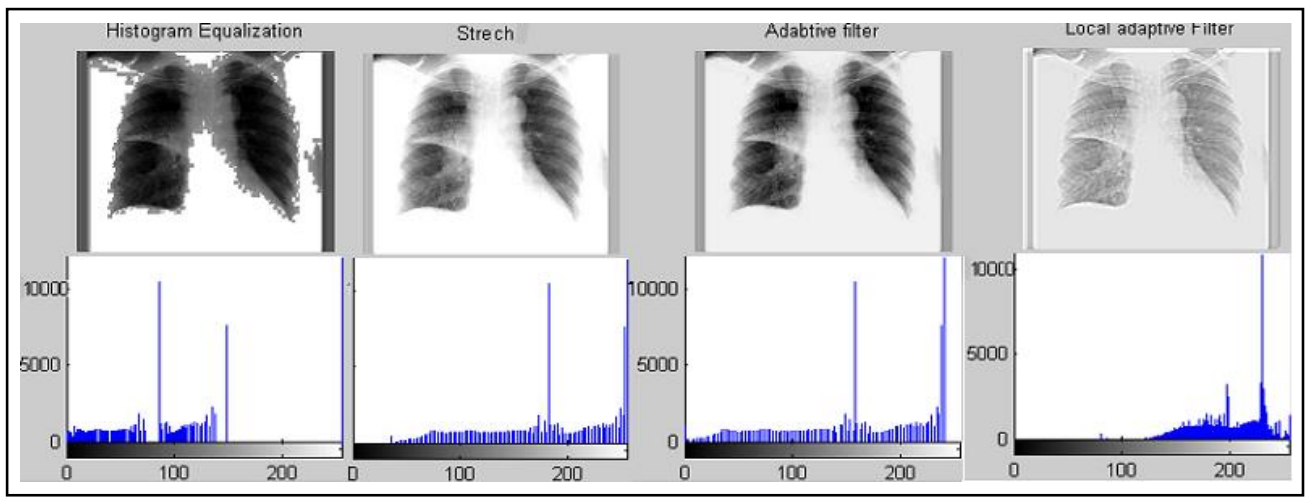

الشكل (10) يوضح الصورة الناتجة من عملية تحسين التباين باستخدام الطرائق التقليدية 
علياء قصي أحمد تقي

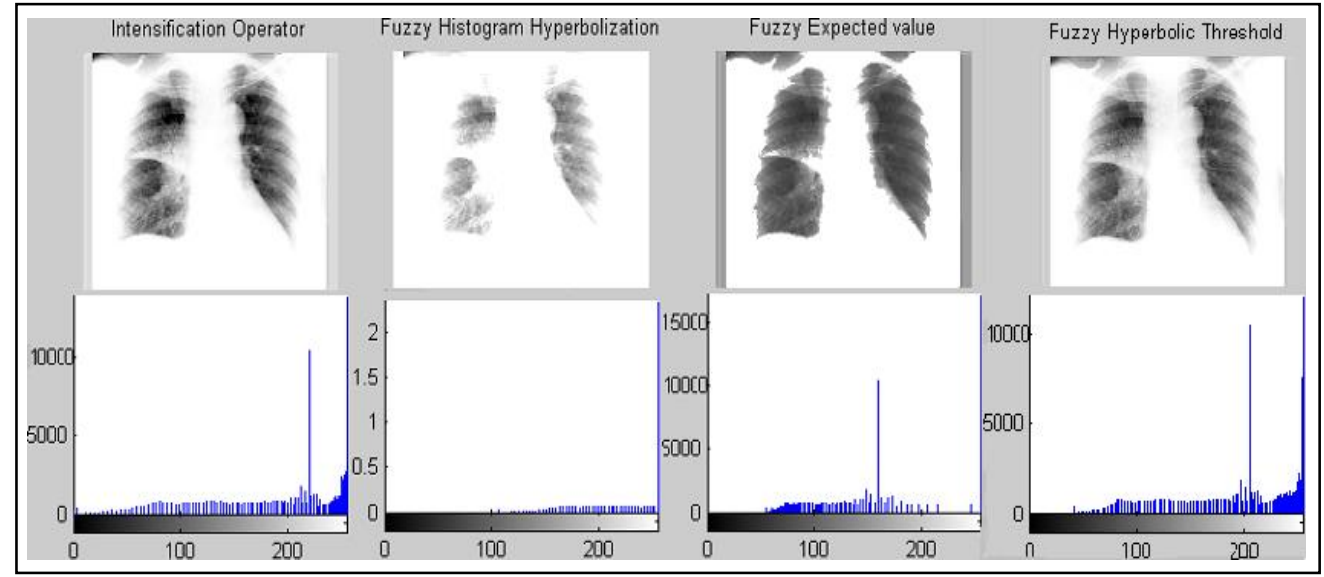

الثكل (11) يوضح الصورة الناتجة من عملية تحسين التباين باستخدام طرائق المنطق المضبب

والثكل الآتي يوضح عملية تحسين التباين لصورة ملونة :

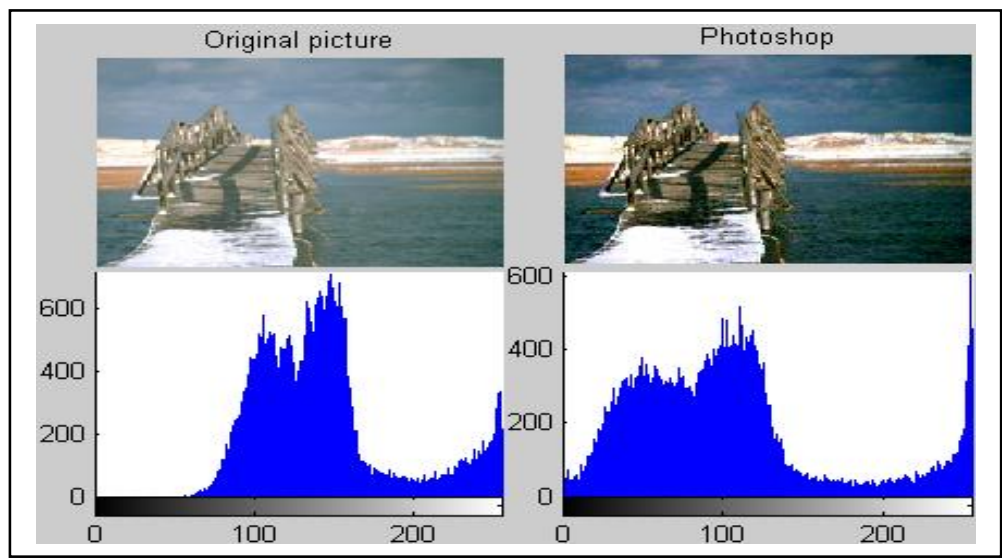

الثكل (12) يوضح الصورة التي ستتم معالجتها والصورة الناتجة من استخدام برنامج (Photoshop)

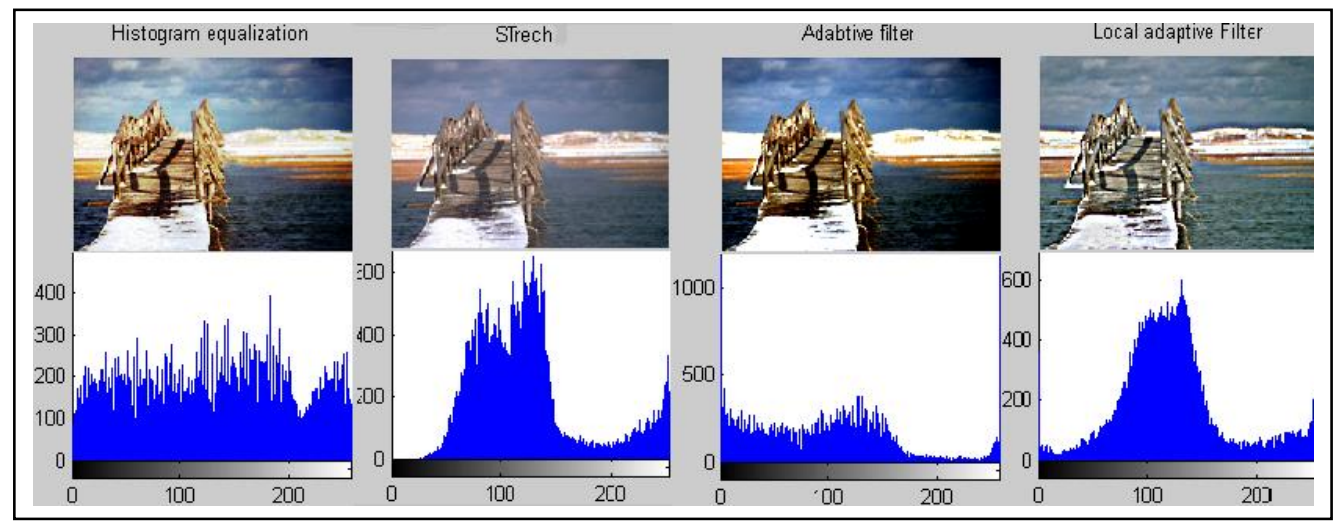

الثكل (13) يوضح الصورة الناتجة من عملية تحسين التباين باستخدام الطرائق التقليدية 


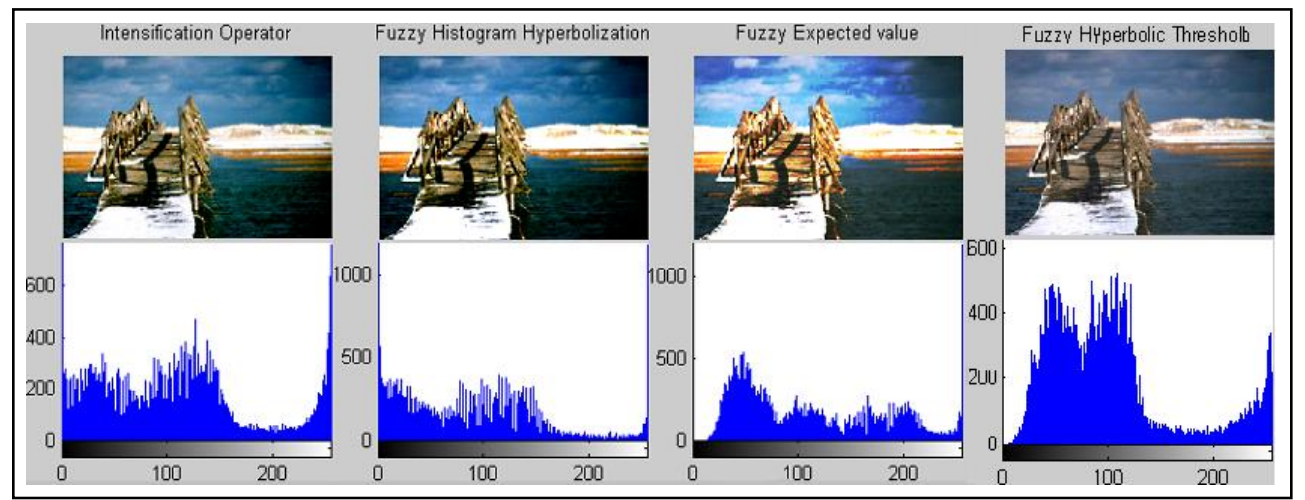

الثكل (14) يوضح الصورة الناتجة من عملية تحسين التباين باستخدام طرائق المنطق المضبب

والثكل الآتي يوضح عملية تحسين التباين على فلم باستخدام طريقة حد العتبة المهنة المضببة:

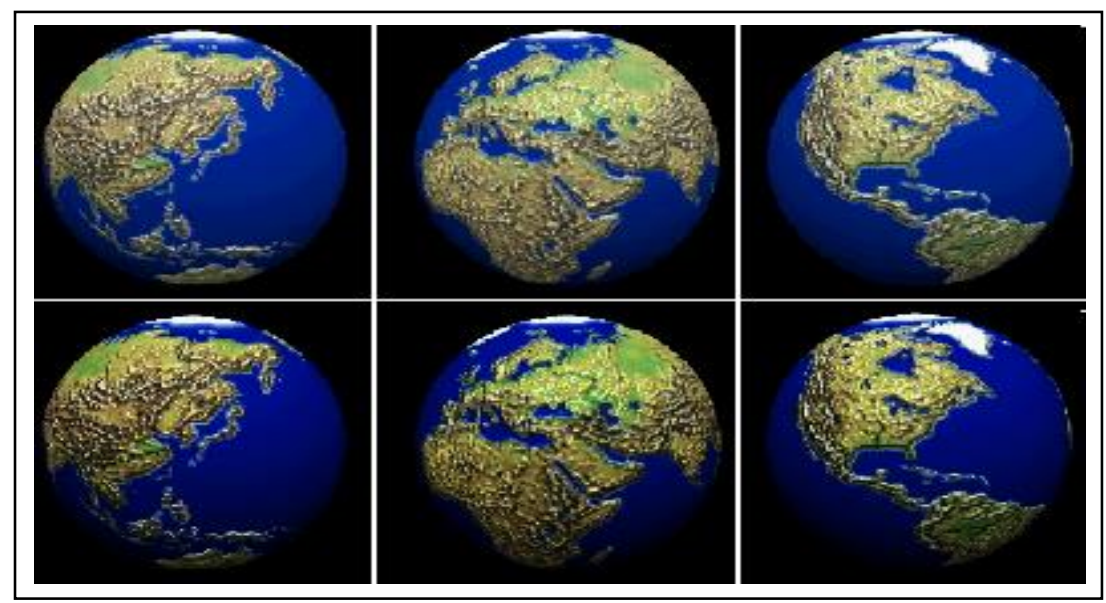

الثكل (15) يوضح الصورة الناتجة من عملية تحسين التباين على فلم

إن مشكلة التباين السيئ موجودة أيضاً في الأفلام الفيديوية، لذا قمنا بتطبيق الطرائق

السابقة على فلم (movie) وقد وحصلنا على نتائج ممتازة إذ يوضح الثكل (15) مقاطع مأخوذة من فلم لدوران الكرة الأرضية، اخترنا منه ثلاث صور ، إذ يتم إجراء المعالجة على الفلم بعد تحويله

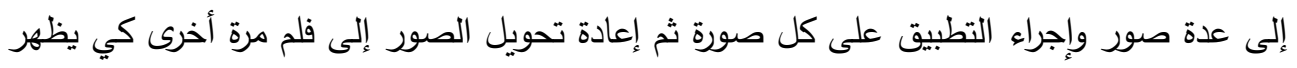

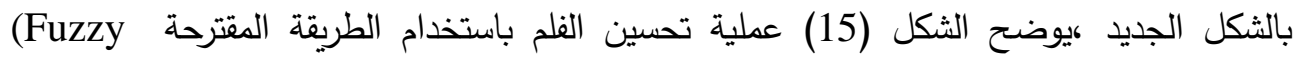
Hyperbolic Threshold)

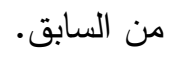


10. الاستنتاجات والتوصيات:

إن التقنيات المستخدمة للتحسين تكون غير موحدة على أنواع الصور كافة فقد تكون الطريقة المستخدمة لتحسين نوع معين لا تعطي أفضل النتائج على نوع آخر وذلك لاختلاف معايير الجودة لكل صورة،فصور التدرج الرمادي تضم صوراً عامة أو صور الأشعة مثلاً وكل منهما له له مواصفات وتركيز في الألوان يختلف عن الآخر أما الصور الملونة فيتم التعامل معها على ألى أنها ثلاث صور ويختلف توزيع كل لون باختلاف الصور للتلك من الصعب تحديد خوارزمية عامة الصاصة

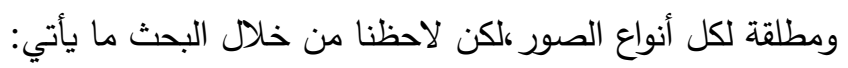

1. إن قياس الأداء لعمليات تحسين التباين في الصور يعتمد على عدة متغيرات مثل قيمة

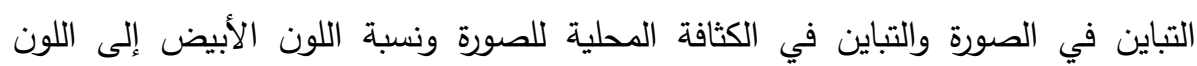

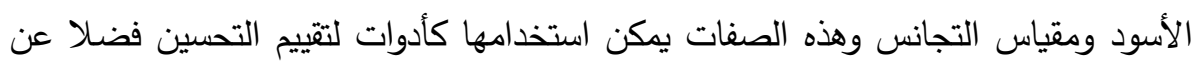

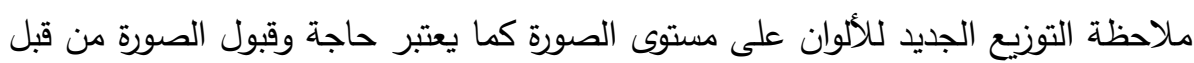
المستخدم أو التطبيق هو المقياس الأفضل.

2. بصورة عامة تكون عمليات التحسين إما عامة (Global Enhancement)، أو الوفيل

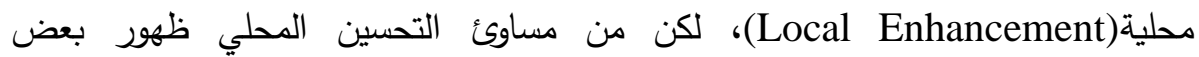

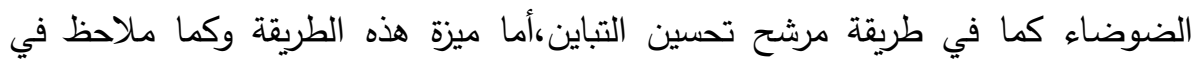

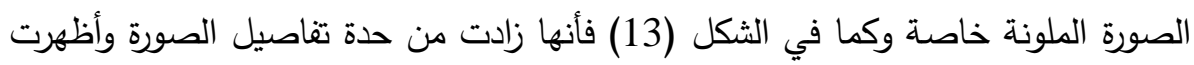
الحواف بشكل جيد كما واضح في أمواج المياه، لذا تعتبر طريقة جيدة لاستخدامها لإبراز

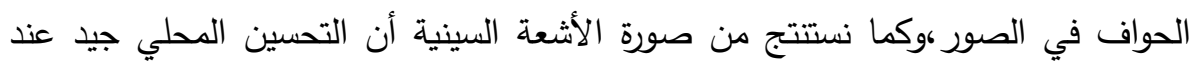

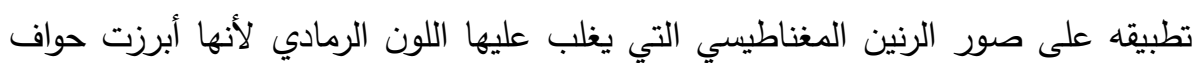
الصورة وحدودها.

3. بالنسبة إلى الطرائق التقليدية فإن طريقة المدرج التكراري المتساوي هي الأقل جودة أما في حالة طرائق المنطق المضبب فكانت طريقة القيمة المضببة المتوقعة هي الأقل جودة ففي كلا الطريقتين فقدت الصورة الكثير من الألوان الأصلية .

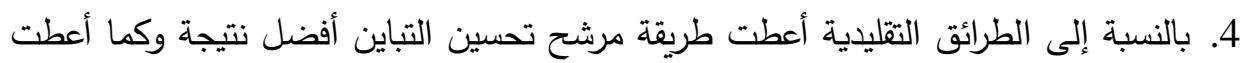
طرنقة نشر القيم نتيجة مقارية للصورة الأصلية، لكن نلاحظ أن الصورة الناتجة معتمة كما

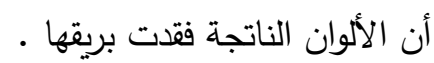
5. أما طرائق المنطق المضبب فتتفاوت جودة النتائج وتوزيع الألوان حسب مواصفات الصورة

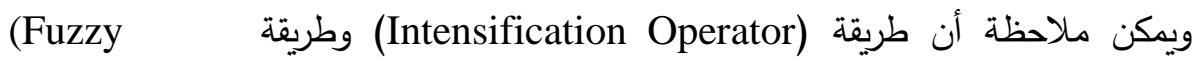


كانتا جيدتين وزادتا من بريق الألوان وأصبحت Histogram Hyperbolization) تفاصيل الصورة أوضح ، لكن حصل فقدان في بعض الألوان، أما الطريقة المقترحة فقد أعطت نتائج جيدة لجميع الصور وكما ملاحظ (Fuzzy Hyperbolic Threshold) من الصور الناتجة ومن توزيع الألوان الناتجة بعد عملية تحسين التباين أن الطريقة المقترحة

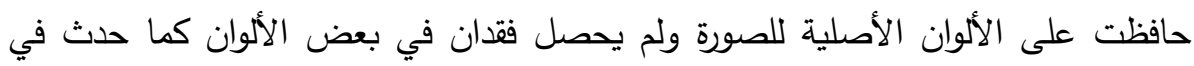

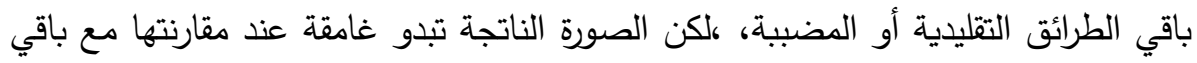

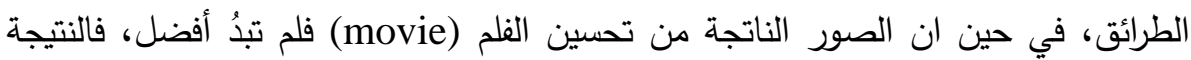
تختلف حسب طبيعة ألوان الصورة. وننصح باستخدام الطريقة المقترحة على الصورة الفاتحة جدا وعلى الصور الطبية لحفاظها على الألوان. 


\section{المصادر}

[1] C.Kotropoulos and I.Pitas,(2001),"Nonlinear model based image/video processing and analysis", A wiley-interscience publication, John wiley \&sous, inc, USA .

[2] Georg J. klir , Ute St.Clair and Bo Yuan,(1997),'Fuzzy set theory Foundations and Applications", Practice Hall PTR,USA.

[3] Haim levkowitz,(1997),'Color theory and modeling for computer Graphics, Visualization, and multimedia Applications ",Kluwer Academic publishers, USA

[4] H.R.Tizhoosh,(1997),Contrast Improvement based with Fuzzy Histogram Hyperbolization”, University of Waterloo, Springer

[5] Jonas Gomes and Luiz Velho,(1997),"image processing for computer Graphics", Springer, New York, New York

[6] Pratt,willeam.K.,(1976),“Digital Image Processing ”,A wileyinterscience publication by John wiley \&sous,inc.

[7] Rafael C.Gonzalez ,Richard E.Woods ,Steven L.Eddins, (2004.), "Digital image Processing using matlab",Prentice Hall, New jersey.

[8] RafaelC.Gonzalez.,Woods,R.E.,(2002), Digital Image Processing ",2nd edition, Publisher Prentic Hal, , New jersey

[9] Tinku acharya and Ajoy k.Ray,(2005),'Image processing Principles and Applications", Prentice Hall, New jersey

[10] Umbaugh Cott E., (1998), "Computer Vision and Image Processing Practical Approach using CVIP tools", Practice Hall PTR. 